\title{
Frame Constructions, Truth Invariance and Validity Preservation in Many-Valued Modal Logic
}

\section{Pantelis E. Eleftheriou $^{1} \quad$ Costas D. Koutras $^{1,2}$}

${ }^{1}$ Graduate Programme in Logic, Algorithms and Computation

M.P.L.A., Dept. of Mathematics

University of Athens

15784 Panepistimioupolis, Ilissia, Greece

${ }^{2}$ Department of Computer Science and Technology

University of Peloponnese

End of Karaiskaki Str., 22100 Tripolis, Greece

e-mail: pelefth@math.uoa.gr

ckoutras@cc.ece.ntua.gr

\begin{abstract}
In this paper we define and examine frame constructions for the family of many-valued modal logics introduced by M. Fitting in the '90s. Every language of this family is built on an underlying space of truth values, a Heyting algebra $\mathcal{H}$. We generalize Fitting's original work by considering complete Heyting algebras as truth spaces and proceed to define a suitable notion of $\mathcal{H}$-indexed families of generated subframes, disjoint unions and bounded morphisms. Then, we provide an algebraic generalization of the canonical extension of a frame and model, and prove a preservation result inspired from Fitting's canonical model argument in [7]. The analog of a complex algebra and of a principal ultrafilter is defined and the embedding of a frame into its canonical extension is presented.
\end{abstract}




\section{Introduction}

Contemporary Modal Logic has been seriously influenced by the applications it has found in Computer Science, Artificial Intelligence, Formal Linguistics and Philosophy $[2,14]$. The key to the success of Modal Logic in so many disciplines is the underlying relational semantics, whose importance for the development of the field can be hardly overstated. The modern trend is to view modal languages as simple languages for talking about relational structures, a formal device that crops over everywhere in Theoretical Computer Science, Knowledge Representation and Cognitive Science. The reader can obtain a picture of the field in the recent books $[2,20,3]$ and the survey article [12].

One of the novel approaches in Modal Logic, rather overlooked hitherto, is the family of many-valued modal logics (mv-MLs) introduced by M. Fitting in a series of papers from 1992 to 1995 [6, 7, 8, 9]. In this approach, Modal Logic is erected on a version of many-valued propositional logic (instead of classical two-valued propositional logic), but in a particular way. Each logic of this family is built on an underlying Heyting algebra $\mathcal{H}$ which provides the space of truth values. Moreover, the elements of $\mathcal{H}$ are directly encoded in the language as special constants and thus we can manipulate them explicitly. Perhaps the most novel feature of this family of logics is the version of relational semantics it possesses: the possible-worlds frames interpreting a language of this family are directed graphs whose edges are labelled with an element of the underlying Heyting algebra $\mathcal{H}$ (henceforth called $\mathcal{H}$-frames). This structure can be conceived as a possible-worlds frame with a many-valued accessibility relation, a special kind of a labelled transition system. Semantically, any complete Heyting algebra can serve as the space of truth values but in order to provide a completeness theorem with respect to a Gentzen-style sequent calculus, a finiteness assumption has been employed by M. Fitting in [7]. Note that this family of logics possesses also a tableau proof procedure [9], modal non-monotonic counterparts $[8,19,16,18]$ and has been recently investigated from the Completeness Theory [17] and the Correspondence Theory perspective [15].

This paper deals with the model theory of the Fitting family of many-valued modal logics, focusing on the 'big four' frame constructions: generated subframes, disjoint unions, bounded morphisms and canonical extensions. Historically, the study of frames has dominated the 'classical era' of Modal Logic (see [2, Ch. 1.7]), during which mainly generated submodels and bounded morphisms were used as tools for transforming the canonical model, in order to prove frame determination results, whenever the 'intoxicatingly successfull' completeness-via-canonicity method failed [2, Ch. 4]. The bounded morphism construction appears usually in the literature as p-morphism, a truncation of pseudo-epimorphism. The subsequent explosion of modal expressivity investigations brought into foreground the constructions of disjoint unions and canonical extensions (also called ultrafilter extensions), which are also useful in another pillar of modal wisdom, the duality theory of Modal Logic (see [12, 2, 20] for results and references). The justification for naming these constructions as the 'big four' constructions in the framebased analysis of modal logic stems partly from the fact that they occur in the celebrated Goldblatt-Thomason theorem [13] which characterizes the elementarily definable classes of frames which are modally definable. Here, we investigate these constructions in the 
context of many-valued modal languages. For the first three cases we can elaborate on $\mathcal{H}$-indexed families of constructions which assert invariance results of a special form: for a fixed truth value $a \in \mathcal{H}$, what remains invariant for a given modal formula inside a state of the modal model is the lattice-theoretic meet of the formula's truth value with $a$. Further on, we proceed to the canonical extension of frames, for languages built on finite Heyting algebras. This construction seems to be the most interesting one, at least mathematically. To define the canonical extension of $\mathcal{H}$-frames and $\mathcal{H}$-models we need to rework many notions, from the algebraic perspective. Building on facts from Universal Algebra, we define the appropriate algebraic analog of a full complex algebra of a frame. Then, we proceed to the associated step of getting the ultrafilter frame of the full complex algebra. The latter step, in the many-valued case employs an involved technical construction inspired from Fitting's canonical model argument in [7]. With the aid of these tools, we obtain an algebraic analog of the classical truth invariance result in the principal ultrafilters of the full complex algebra of a modal frame. En route, we discuss also the related areas of algebraic semantics and general $\mathcal{H}$-frames for these languages. Finally, we hint on the notion of bisimulation, which is very important at the model level.

In Section 2 we provide the mathematical background and in Section 3 the syntax and semantics of many-valued modal languages, including an alternative multiple-expert semantics that may be of some interest in Knowledge Representation situations. Section 4 contains the results of this paper, treating the four frame and model constructions for many-valued modal languages. The paper ends in Section 5 with a discussion of our results.

\section{Mathematical Preliminaries}

This section serves mainly for establishing notation and terminology and for recording some facts that we will use in the proofs of our results. We assume the reader is well acquainted with the theory of Heyting Algebras (henceforth HAs) and their properties. For details and proofs see the classical texts [21, 1]; [23] is useful for the elements of Universal Algebra. In a lattice $\langle L, \leq\rangle$, we denote the least upper bound or join, by $a+b$, and the greatest lower bound or meet by $a \cdot b$. A least (or bottom) element of a lattice is denoted by $\perp$ and a greatest (or top) one by $T$. Some fairly obvious properties of infinite joins and meets, such as $\prod_{t \in T}\left(a \cdot a_{t}\right)=a \cdot \prod_{t \in T} a_{t}$ will be used, generally without comment. The operation of relative pseudo-complementation is denoted by $a \Rightarrow b$ and its existence in a lattice is equivalent to the fundamental property $\mathcal{H}_{1}: x \leq(a \Rightarrow b)$ iff $(x \cdot a) \leq b$. The following properties of HAs will be used in our proofs: $\mathcal{H}_{2}:(a \Rightarrow b)=\top$ iff $a \leq b$, $\mathcal{H}_{3}:(\top \Rightarrow a)=a, \mathcal{H}_{4}: a_{1} \leq a_{2}$ implies $\left(a_{2} \Rightarrow b\right) \leq\left(a_{1} \Rightarrow b\right), \mathcal{H}_{5}: b_{1} \leq$ $b_{2}$ implies $\left(a \Rightarrow b_{1}\right) \leq\left(a \Rightarrow b_{2}\right), \mathcal{H}_{6}:(a \Rightarrow b) \cdot(a \Rightarrow c)=(a \Rightarrow b \cdot c), \mathcal{H}_{7}:(a \Rightarrow$ $c) \cdot(b \Rightarrow c)=((a+b) \Rightarrow c), \mathcal{H}_{8}: a \Rightarrow(b \Rightarrow c)=(a \cdot b) \Rightarrow c=b \Rightarrow(a \Rightarrow c)$, $\mathcal{H}_{9}: a \cdot(a \Rightarrow b)=(a \cdot b) \leq b, \mathcal{H}_{10}: c \cdot(a \Rightarrow b)=c \cdot((c \cdot a) \Rightarrow(c \cdot b))$, $\mathcal{H}_{11}: a \leq((a \Rightarrow b) \Rightarrow b)$. Also, provided that the (possibly infinite) meets and joins 
exist, $\mathcal{H}_{1}^{\Pi}: \prod_{t \in T}\left(a \Rightarrow b_{t}\right)=\left(a \Rightarrow \prod_{t \in T} b_{t}\right), \mathcal{H}_{1}^{\Pi}: \prod_{t \in T}\left(a \Rightarrow b_{t}\right)=\left(a \Rightarrow \prod_{t \in T} b_{t}\right)$ and $\mathcal{H}_{1}^{\Pi}: \prod_{t \in T}\left(a \Rightarrow b_{t}\right)=\left(a \Rightarrow \prod_{t \in T} b_{t}\right)$.

\section{Syntax and Semantics of Many-Valued Modal Logic}

The syntax of a propositional many-valued modal language is defined by first fixing a complete $\mathrm{HA} \mathcal{H}$ which will serve as the space of truth values. The elements of $\mathcal{H}$ are directly represented in the language by special propositional constants. The syntax definition proceeds as in the classical case, with the remark that the propositional connectives $\vee, \wedge, \supset$ need to be explicitly introduced, as they are no more interdefinable (negation is non-classical); similarly for the two modal operators. In the BNF specification below, a ranges over elements of $\mathcal{H}, P$ ranges over elements of a countably infinite set of propositional variables $\Phi$ and $A$ is a formula of $L_{\square \diamond}^{\mathcal{H}}(\Phi)$.

$$
A::=a|P| A_{1} \vee A_{2}\left|A_{1} \wedge A_{2}\right| A_{1} \supset A_{2}|\square A| \diamond A
$$

$\left(A_{1} \equiv A_{2}\right)$ abbreviates $\left(A_{1} \supset A_{2}\right) \wedge\left(A_{2} \supset A_{1}\right)$ and $\neg A$ abbreviates $(A \supset \perp)$.

Now, we turn to the semantics of many-valued modal languages. The semantics described below alters in a very interesting manner the notion of relational frames, in that it concerns a kind of many-valued accessibility relation.

Definition 3.1 An $\mathcal{H}$-modal frame for $L_{\square \diamond}^{\mathcal{H}}(\Phi)$ is a pair $\mathfrak{F}=\langle\mathfrak{S}, \mathfrak{g}\rangle$, where $\mathfrak{S}$ is a nonempty set of states and $\mathfrak{g}: \mathfrak{S} \times \mathfrak{S} \rightarrow \mathcal{H}$ is a total function mapping pairs of states to elements of $\mathcal{H}$.

An $\mathcal{H}$-modal model $\mathfrak{M}=\langle\mathfrak{S}, \mathfrak{g}, v\rangle$ is built on $\mathfrak{F}$ by providing a valuation $v$, that is a function $v: \mathfrak{S} \times(\mathcal{H} \cup \Phi) \rightarrow \mathcal{H}$ which assigns an $\mathcal{H}$-truth value to atomic formulas in each state, such that $v(\mathfrak{s}, a)=a$, for every $\mathfrak{s} \in \mathfrak{S}$ and $a \in \mathcal{H}$. That is, propositional constants are always mapped to 'themselves'. Valuation extends to all formulae in a standard recursive fashion. The propositional connectives are interpeted over the corresponding algebraic operation: $v(\mathfrak{s}, A \wedge B)=v(\mathfrak{s}, A) \cdot v(\mathfrak{s}, B), v(\mathfrak{s}, A \vee B)=v(\mathfrak{s}, A)+v(\mathfrak{s}, B)$, $v(\mathfrak{s}, A \supset B)=v(\mathfrak{s}, A) \Rightarrow v(\mathfrak{s}, B)$, while for the modal operators

$$
\begin{aligned}
v(\mathfrak{s}, \square A) & =\prod_{\mathfrak{t} \in \mathfrak{S}}(\mathfrak{g}(\mathfrak{s}, \mathfrak{t}) \Rightarrow v(\mathfrak{t}, A)) \\
\text { - } v(\mathfrak{s}, \diamond A) & =\sum_{\mathfrak{t} \in \mathfrak{S}}(\mathfrak{g}(\mathfrak{s}, \mathfrak{t}) \cdot v(\mathfrak{t}, A))
\end{aligned}
$$

Definition 3.2 A formula $A$ of $L_{\square \diamond}^{\mathcal{H}}$ is true in a state $\mathfrak{s}$ of an $\mathcal{H}$-model $\mathfrak{M}=\langle\mathfrak{S}, \mathfrak{g}, v\rangle$ (denoted as $\mathfrak{M}, \mathfrak{s} \Vdash A$ ) if $v(\mathfrak{s}, A)=\top$; we also say that $A$ is satisfied in $\mathfrak{s}$. Further on, $A$ is true in a model $\mathfrak{M}$ (denoted as $\mathfrak{M} \Vdash A$ ) if $v(\mathfrak{t}, A)=\top$ for every $\mathfrak{t} \in \mathfrak{S}$, and it is valid in an $\mathcal{H}$-frame $\mathfrak{F}$ (denoted as $\mathfrak{F} \Vdash A$ ) if it is true in every $\mathcal{H}$-model built on $\mathfrak{F}$. The notions of truth and validity extend in a class $\mathrm{C}$ of $\mathcal{H}$-models or $\mathcal{H}$-frames, in the obvious way (notation: $\mathrm{C} \Vdash A$ ). 
Finally, a modal formula $A$ is called a-true in an $\mathcal{H}$-model $\mathfrak{M}$, if the formula $(a \supset A)$ is true in $\mathfrak{M}$; similarly for $a$-validity. We assume that the reader is acquainted with the notion of correspondence between a modal formula and a class of frames (see [2]).

\subsection{Multiple-Expert Semantics for many-valued modal languages}

Let us, for the moment, confine ourselves in the class of many-valued modal languages built on finite HAs. Assume an epistemic situation involving (possibly infinitely) many states of affairs (possible worlds) and (finitely) many experts, whose opinion we value. Obviously, if the experts are independent there is nothing genuinely interesting to deal with. The scenario becomes interesting when the experts are related through a sort of hierarchical organization, which affects the way each of them makes up her mind on the truth of certain statements: some experts dominate others. Reasonably enough, we consider that the binary dominance relation is antisymmetric and transitive, and is broadly conceived: every experts dominates herself, that is the relation is also reflexive. In simple words, a partial order relation is imposed on the experts. Before proceeding to a technical description of the epistemic situations, an example is in order.

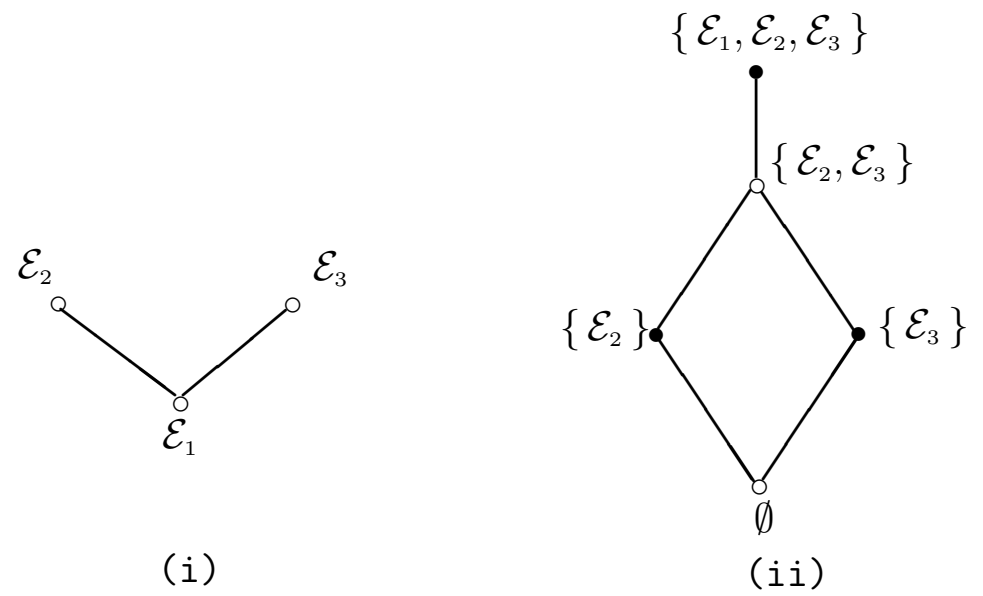

Figure 1: From many experts (i), to many truth values (ii), and vice versa

Assume we have three experts $\mathcal{E}_{1}, \mathcal{E}_{2}, \mathcal{E}_{3}$, related as in Fig. 1.(i): $\mathcal{E}_{1}$ dominates $\mathcal{E}_{2}$ and $\mathcal{E}_{3}{ }^{1}$. Further on, assume a set of possible worlds, common to all agents and a common epistemic language. We require that the dominance relation has the following effect on the accessibility relation each expert considers on the set of possible worlds:

$\mathrm{D}_{1}$ : an expert $\mathcal{E}_{i}$ considers that $\mathfrak{s}_{1}$ 'sees' $\mathfrak{s}_{2}$, iff every expert dominated by $\mathcal{E}_{i}$, does so

Note that each expert provides us a classical relational frame but, as a whole, the experts' accessibility relations should satisfy condition $D_{1}$. We can proceed from multiple-expert

\footnotetext{
${ }^{1}$ It would be perhaps more intuitive to represent the hierarchically ordered experts, by placing the 'dominating' above the 'dominated' ones. We have chosen however to follow the standard mathematical practice and represent both the partial ordering of Fig. 1.(i), as well as the lattice of Fig. 1.(ii) with their Hasse diagrams; this will facilitate the discussion on the transitions between these two structures.
} 
frames to models, by providing a valuation. Again, we impose a similar requirement, which reasonably reflects the effect of dominance in truth assignments to propositional variables, for every expert, inside every possible world:

\section{$\mathrm{D}_{2}$ : what an expert $\mathcal{E}_{i}$ accepts to be true in a possible world, must also be accepted by every expert $\mathcal{E}_{i}$ dominates}

The rules for extending the valuation to all formulae of the epistemic language, also reflect the dominance relation. Formulae of the form $A \wedge B$ and $A \vee B$ are treated locally by an expert inside a possible world, in the obvious way. On the other hand, formulae of the form $\neg A, A \supset B, \square A, \diamond A$, are set to true by an expert inside a possible world, iff every dominated agent considers them to be true also in this possible world. A flavour of intuitionistic logic is more than obvious.

We are interested now in finding the experts' 'consensus', that is, in elegantly calculating the modal formulae on which our experts agree. This problem can be reformulated as one involving a many-valued language, where sets of experts who agree on the truth of an epistemic statement can be seen as generalized truth values. For instance, to denote that all experts agree on the truth of $\square A$, we say that the truth value of $\square A$ is $\left\{\mathcal{E}_{1}, \mathcal{E}_{2}, \mathcal{E}_{3}\right\}$. Note however an important point: by $\mathrm{D}_{2}$, not every set of experts is an 'admissible' generalized truth value. For instance, it cannot happen that $\mathcal{E}_{1}$ alone considers $A$ to be true. The 'admissible' sets of experts are those which are dominance-closed, that is, upwards-closed in ordered sets terminology. Similarly for the accessibility relation between states: it can be given a generalized truth value (the set of experts who agree on the existence of an edge) and 'admits' (by $D_{1}$ ) the same sets of experts as such. To complete the example, it remains to notice that the 'admissible' sets of experts provide us a finite Heyting algebra, when ordered under set inclusion: the lattice of sets of Fig. 1.(ii). A straightforward proof verifies that the rules (we informally sketched above) for extending the valuation to all formulae preserve requirement $\mathrm{D}_{2}$, and thus this kind of multiple-expert modal models 'captures' the dominance relation. Actually, the multiple-expert situation described can be entirely translated to a many-valued modal model of the language $L_{\square \diamond}^{\mathcal{H}}$, where $\mathcal{H}$ is the lattice of Fig. 1.(ii); for a detailed proof of this claim see [7, Sect. 5]. The other translation is also feasible: as a first step, the reader can check that we recover the dominance relation of Fig. 1.(i) by ordering (under set inclusion) the prime filters of the lattice in Fig. 1.(ii) ${ }^{2}$.

In general, multiple-expert epistemic situations of the kind sketched above are formally described by multiple-expert modal frames of the form $\langle\mathcal{E}, \mathcal{D}, \mathcal{G}, \mathcal{R}\rangle$, where: $\mathcal{E}$ is a finite non-empty set of experts, $\mathcal{D}$ is the partial-order dominance relation on $\mathcal{E}, \mathcal{G}$ is a non-empty set of possible worlds, and $\mathcal{R}$ is an $\mathcal{E}$-indexed set of accessibility relations on $\mathcal{G}$ satisfying requirement $\mathrm{D}_{1}$ [7, Sect. 3]. We construct a multiple-expert modal model by providing a valuation $v$ that respects $\mathrm{D}_{2}$, and in a similar fashion, we extend it to all modal formulae (as sketched above). In [7] the equivalence of multiple-expert modal models with the $\mathcal{H}$-modal models is established and thus we speak of an equivalent formulation of these logics. The starting point for proving this equivalence is actually

\footnotetext{
${ }^{2}$ Note that in finite HAs, prime filters are obtained as the principal filters generated by the joinirreducible elements [5, Lem. 8.16, Prop. 9.4]. The join-irreducible elements of the lattice in Fig. 1.(ii) are shaded.
} 
G. Birkhoff's theorem on the dual representation between finite partial orders and finite distributive lattices (that is, finite HAs), as in our example above; see [5, 8.17-8.19] for a proof stated in terms of down-sets and prime ideals. In [7] this is significantly extended, actually lifting to the modal setting the proofs on the equivalence between the algebraic and the possible- world semantics of propositional intuitionistic logic. The technical construction is very elegant and at the same time a very interesting example of application of mathematical ideas in AI problems.

The interested reader should verify, after reading the next section where our results are exhibited, that we proceed to define model theoretic construction which guarantee to keep invariant the epistemic consensus of a predefined group of experts. Thus, they might be of some interest to the Knowledge Representation community. Note though, that our results carried out in the many-valued facet of these logics are more general as they hold for any complete Heyting algebra.

\section{Model and Frame Constructions in mv-ML}

\subsection{Generated Subframes and Submodels}

In the classical case, generated submodels embody a facet of the 'locality of computation' in relational semantics. Namely, the fact that in order to compute the truth value of any modal formula in a state $s$ of a model, we only need the set of states that can be accessed from s following any finite number of $\mathcal{R}$-steps, where $\mathcal{R}$ is the binary accessibility relation. This allows to prove a truth invariance result and a validity preservation result. The former, at the level of modal models, states that the modal theory of a state in a model coincides with its modal theory in the model this state 'generates'.

In many-valued modal logic, we can refine this idea to a more general one. For every truth value $a \in \mathcal{H}$ and a set of states, we build a 'generated' subframe in which we keep only those edges whose label does not provide the $\perp$ element as the meet with $a$. For 'generated' submodels, we further require that the assignment of truth values in the two models, satisfies a similar requirement ${ }^{3}$. Below, we prove that this allows us to prove an $a$-invariance result at the model level.

Definition 4.1 Let $\mathfrak{M}=\langle\mathfrak{S}, \mathfrak{g}, v\rangle$ and $\mathfrak{M}^{\prime}=\left\langle\mathfrak{S}^{\prime}, \mathfrak{g}^{\prime}, v^{\prime}\right\rangle$ be two $\mathcal{H}$-models for $L_{\square \diamond}^{\mathcal{H}}(\Phi)$. $\mathfrak{M}^{\prime}$ is an a-preserving generated submodel of $\mathfrak{M}$ (notation: $\mathfrak{M}^{\prime} \longmapsto^{a} \mathfrak{M}$ ) iff:

1. $\mathfrak{S}^{\prime} \subseteq \mathfrak{S}$

2. for states $\mathfrak{s}, \mathfrak{t}$ in $\mathfrak{S}^{\prime}: \quad a \cdot \mathfrak{g}^{\prime}(\mathfrak{s}, \mathfrak{t})=a \cdot \mathfrak{g}(\mathfrak{s}, \mathfrak{t})$

3. for every $\mathfrak{s} \in \mathfrak{S}^{\prime}$ and $P \in \Phi: \quad a \cdot v^{\prime}(\mathfrak{s}, P)=a \cdot v(\mathfrak{s}, P)$

\footnotetext{
${ }^{3}$ Actually, the term inner substructure, used in $[11,12]$, would be more appropriate here, as there is no 'generation' actually involved. We decided however to stay with the more standard terminology of generated subframes and submodels.
} 
and the following closure condition holds:

4. if $\mathfrak{s} \in \mathfrak{S}^{\prime}$ and $a \cdot \mathfrak{g}(\mathfrak{s}, \mathfrak{t}) \neq \perp$, then $\mathfrak{t} \in \mathfrak{S}^{\prime} \quad(a-c c)$

The notion of an a-preserving generated subframe $\mathfrak{F}^{\prime}=\left\langle\mathfrak{S}^{\prime}, \mathfrak{g}^{\prime}\right\rangle$ of $\mathfrak{F}=\langle\mathfrak{S}, \mathfrak{g}\rangle$ (notation: $\left.\mathfrak{F}^{\prime} \longmapsto^{a} \mathfrak{F}\right)$ is defined by leaving out item (3).

The following theorem states that modal truth is $a$-invariant under $a$-preserving generated submodels.

Theorem 4.2 For $\mathcal{H}$-models $\mathfrak{M}=\langle\mathfrak{S}, \mathfrak{g}, v\rangle$ and $\mathfrak{M}^{\prime}=\left\langle\mathfrak{S}^{\prime}, \mathfrak{g}^{\prime}, v^{\prime}\right\rangle$, let $\mathfrak{M}^{\prime} \longmapsto^{a} \mathfrak{M}$. Then, for each formula $A$ of $L_{\square \diamond}^{\mathcal{H}}$ and state $\mathfrak{s}$ of $\mathfrak{M}^{\prime}$,

$$
a \cdot v(\mathfrak{s}, A)=a \cdot v^{\prime}(\mathfrak{s}, A)
$$

Proof. By induction on $A$. The case of propositional variables and constants is trivial. The argument for the propositional connectives employs the properties of HAs; for instance the proof for disjunction requires just the distributivity of HAs and implication requires property $\mathcal{H}_{10}$; the reader can easily verify. We provide the argument for $\square$ and note that the argument for $\diamond$ is similar.

$$
\begin{aligned}
& a \cdot v\left(\mathfrak{s}, \square A_{1}\right) \quad=\quad a \cdot \prod_{\mathfrak{t} \in \mathfrak{S}}\left(\mathfrak{g}(\mathfrak{s}, \mathfrak{t}) \Rightarrow v\left(\mathfrak{t}, A_{1}\right)\right) \\
& =\prod_{\mathfrak{t} \in \mathfrak{S}}\left(a \cdot\left(\mathfrak{g}(\mathfrak{s}, \mathfrak{t}) \Rightarrow v\left(\mathfrak{t}, A_{1}\right)\right)\right) \\
& =\prod_{\mathfrak{t} \in \mathfrak{S}} a \cdot\left(a \cdot \mathfrak{g}(\mathfrak{s}, \mathfrak{t}) \Rightarrow a \cdot v\left(\mathfrak{t}, A_{1}\right)\right) \quad\left(\text { by } \mathcal{H}_{10}\right) \\
& =\prod_{\mathfrak{t} \in \mathfrak{S}^{\prime}} a \cdot\left(a \cdot \mathfrak{g}(\mathfrak{s}, \mathfrak{t}) \Rightarrow a \cdot v\left(\mathfrak{t}, A_{1}\right)\right) \quad \text { (by } \mathcal{H}_{1} \& \text { Def. 4.1, a-cc) } \\
& =\prod_{\mathfrak{t} \in \mathfrak{S}^{\prime}} a \cdot(\underbrace{a \cdot \mathfrak{g}^{\prime}(\mathfrak{s}, \mathfrak{t})}_{\text {by Def. 4.1 }} \Rightarrow \underbrace{a \cdot v^{\prime}\left(\mathfrak{t}, A_{1}\right)}_{\text {Induct. Hypothesis }}) \\
& =a \cdot \prod_{\mathfrak{t} \in \mathfrak{S}^{\prime}}\left(\mathfrak{g}^{\prime}(\mathfrak{s}, \mathfrak{t}) \Rightarrow v^{\prime}\left(\mathfrak{t}, A_{1}\right)\right) \quad\left(\text { by } \mathcal{H}_{10}\right) \\
& =a \cdot v^{\prime}\left(\mathfrak{s}, \square A_{1}\right)
\end{aligned}
$$

The frame validity preservation result, whose proof is easy, is as follows:

Theorem 4.3 Let $\mathfrak{F}=\langle\mathfrak{S}, \mathfrak{g}\rangle$ be an $\mathcal{H}$-frame. For every $X \in L_{\square \diamond}^{\mathcal{H}}$,

$$
\mathfrak{F} \Vdash_{a} X \text { iff for every } \mathcal{H} \text {-frame } \mathfrak{F}^{\prime}, \quad \mathfrak{F}^{\prime} \longmapsto^{a} \mathfrak{F} \text { implies } \mathfrak{F}^{\prime} \Vdash_{a} X
$$




\subsection{Disjoint Unions}

The construction of disjoint unions is of interest mainly to the definability theory of modal logic. Its direct transfer to many-valued modal logic is more or less immediate, in the sense that, again, computation is 'local': the truth value of a formula is not affected by inaccessible worlds and disconnected components of the edge-labelled directed graph. Now, in the context we examine here, we can provide a fairly general notion of a disjoint union which allows us to prove an $a$-invariance result, as in the previous subsection.

Definition 4.4 For an index set $I$, let $\mathfrak{M}_{i}=\left\langle\mathfrak{S}_{i}, \mathfrak{g}_{i}, v_{i}\right\rangle,(i \in I)$ be a collection of disjoint (for every $\left.i, j \in I, \mathfrak{S}_{i} \cap \mathfrak{S}_{j}=\emptyset\right) \mathcal{H}$-models for $L_{\square \diamond}^{\mathcal{H}}(\Phi)$. The a-preserving disjoint union of this collection, is the $\mathcal{H}$-model $\biguplus_{i} \mathfrak{M}_{i}=\langle\mathfrak{S}, \mathfrak{g}, v\rangle$, where

1. $\mathfrak{S}$ is the union of the sets $\mathfrak{S}_{i}$

2. for every $\mathfrak{s} \in \mathfrak{S}_{i}, \mathfrak{t} \in \mathfrak{S}_{j}: a \cdot \mathfrak{g}(\mathfrak{s}, \mathfrak{t})=a \cdot \mathfrak{g}_{i}(\mathfrak{s}, \mathfrak{t})$ if $i=j$, else $a \cdot \mathfrak{g}(\mathfrak{s}, \mathfrak{t})=\perp$

3. for every $\mathfrak{s} \in \mathfrak{S}_{i}$ and $P \in \Phi, a \cdot v(\mathfrak{s}, P)=a \cdot v_{i}(\mathfrak{s}, P)$

The $a$-preserving disjoint union of $\mathcal{H}$-frames $\biguplus_{i} \mathfrak{F}_{i}=\langle\mathfrak{S}, \mathfrak{g}\rangle$ is defined by leaving out item (3).

The truth invariance result is stated in the next theorem: modal truth is $a$-invariant under $a$-disjoint unions. The proof runs by induction and requires property $\mathcal{H}_{1}^{\sum}$; it is left to the reader.

Theorem 4.5 Let $\biguplus_{i} \mathfrak{M}_{i}=\langle\mathfrak{S}, \mathfrak{g}, v\rangle$ be the disjoint union of a collection of disjoint $\mathcal{H}$-models $\mathfrak{M}_{i}=\left\langle\mathfrak{S}_{i}, \mathfrak{g}_{i}, v_{i}\right\rangle,(i \in I)$. Then, for each formula $A$ of $L_{\square \diamond}^{\mathcal{H}}$ and state $\mathfrak{s}$ of $\mathfrak{M}_{i}$,

$$
a \cdot v(\mathfrak{s}, A)=a \cdot v_{i}(\mathfrak{s}, A) .
$$

Using the results we have proved so far, we can prove that naturally arising logical modalities, variants of the global modality and the difference operator (see [2, Ch. 7]) are not definable in the language $L_{\square \diamond}^{\mathcal{H}}$. For instance, it is natural to consider a global modality $\mathbf{A}_{a} \varphi(a \in \mathcal{H})$ which would capture the fact that $\varphi$ has the truth value $a$ in every state of the $\mathcal{H}$-model considered. A 'dual' existential modality $\mathbf{E}_{a} \varphi$ can be also considered. A variant of the difference operator is given below: $\mathbf{D}_{a} \varphi$ is true in a state $\mathfrak{s}$, iff $\varphi$ has the truth value $a$ in a different state $\mathfrak{t}$. None of these modalities is $L_{\square \diamond}^{\mathcal{H}}$-definable. The proof is easy and it is left to the reader.

Example 4.6 None of the following logical modalities is definable in $L_{\square \diamond}^{\mathcal{H}}$.

- $v\left(\mathfrak{s}, \mathbf{A}_{a} \varphi\right)=\top \quad$ iff $\quad v(\mathfrak{t}, \varphi)=a$, for every $\mathfrak{t} \in \mathfrak{S}$

- $v\left(\mathfrak{s}, \mathbf{E}_{a} \varphi\right)=\top \quad$ iff $\exists \mathfrak{t} \in \mathfrak{S}$ s.t. $v(\mathfrak{t}, \varphi)=a$

- $v\left(\mathfrak{s}, \mathbf{D}_{a} \varphi\right)=\top \quad$ iff $\exists \mathfrak{t} \in \mathfrak{S}$ s.t. $\mathfrak{t} \neq \mathfrak{s}$, and $v(\mathfrak{t}, \varphi)=a$ 
The validity preservation principle at the level of $\mathcal{H}$-frames is given in the next theorem.

Theorem 4.7 Let $\biguplus_{i} \mathfrak{F}_{i}=\langle\mathfrak{S}, \mathfrak{g}\rangle$ be the disjoint union of a collection of disjoint $\mathcal{H}$ frames $\mathfrak{F}_{i}=\left\langle\mathfrak{S}_{i}, \mathfrak{g}_{i}\right\rangle,(i \in I)$. Then, for each formula $X$ of $L_{\square \diamond}^{\mathcal{H}}$,

$$
\biguplus_{i} \mathfrak{F}_{i} \Vdash_{a} X \quad \text { iff } \forall i \in I, \mathfrak{F}_{i} \Vdash_{a} X
$$

\subsection{Bounded Morphisms}

The case of bounded morphisms is a bit more interesting, technically. We again define a family of transformations that guarantee an $a$-invariance result. Note below however, that the traditional back and forth conditions need not be in absolute 'symmetry'. This will be of help in cases, such as the one in Example 4.11.

Definition 4.8 Let $\mathfrak{M}=\langle\mathfrak{S}, \mathfrak{g}, v\rangle$ and $\mathfrak{M}^{\prime}=\left\langle\mathfrak{S}^{\prime}, \mathfrak{g}^{\prime}, v^{\prime}\right\rangle$ be two $\mathcal{H}$-models for $L_{\square \diamond}^{\mathcal{H}}(\Phi)$. An a-preserving bounded $\mathcal{H}$-morphism from $\mathfrak{M}$ to $\mathfrak{M}^{\prime}$ is a function $f: \mathfrak{S} \rightarrow \mathfrak{S}^{\prime}$ satisfying the following conditions, where $\mathfrak{s}, \mathfrak{t} \in \mathfrak{S}$ are states of $\mathfrak{M}$ and $P \in \Phi$ is a propositional variable:

1. $a \cdot v(\mathfrak{s}, P)=a \cdot v^{\prime}(f(\mathfrak{s}), P)$

2. $a \cdot \mathfrak{g}(\mathfrak{s}, \mathfrak{t}) \leq a \cdot \mathfrak{g}^{\prime}(f(\mathfrak{s}), f(\mathfrak{t})) \quad$ (forth)

3. for every $\mathfrak{s} \in \mathfrak{S}$ and $\mathfrak{u}^{\prime} \in \mathfrak{S}^{\prime}: \quad$ (back) if $a \cdot \mathfrak{g}^{\prime}\left(f(\mathfrak{s}), \mathfrak{u}^{\prime}\right) \neq \perp$,

then $\exists \mathfrak{u} \in \mathfrak{S}$ such that $a \cdot \mathfrak{g}(\mathfrak{s}, \mathfrak{u})=a \cdot \mathfrak{g}^{\prime}\left(f(\mathfrak{s}), \mathfrak{u}^{\prime}\right)$ and $f(\mathfrak{u})=\mathfrak{u}^{\prime}$

For $\mathfrak{F}=\langle\mathfrak{S}, \mathfrak{g}\rangle$ and $\mathfrak{F}^{\prime}=\left\langle\mathfrak{S}^{\prime}, \mathfrak{g}^{\prime}\right\rangle \mathcal{H}$-modal frames, an a-preserving bounded $\mathcal{H}$-morphism from $\mathfrak{F}$ to $\mathfrak{F}^{\prime}$ is a function $f: \mathfrak{S} \rightarrow \mathfrak{S}^{\prime}$ which satisfies the above (forth) and (back) conditions. If $f$ is onto, we call $\mathfrak{F}^{\prime}\left(\mathfrak{M}^{\prime}\right)$ an $a$-preserving bounded morphic image of $\mathfrak{F}$ $(\mathfrak{M})$ through $f$ (notation: $\mathfrak{F} \rightarrow^{a} \mathfrak{F}^{\prime}\left(\mathfrak{M} \rightarrow{ }^{a} \mathfrak{M}^{\prime}\right)$ ).

Theorem 4.9 Let $\mathfrak{M}=\langle\mathfrak{S}, \mathfrak{g}, v\rangle$ and $\mathfrak{M}^{\prime}=\left\langle\mathfrak{S}^{\prime}, \mathfrak{g}^{\prime}, v^{\prime}\right\rangle$ be two $\mathcal{H}$-models and $f: \mathfrak{S} \rightarrow \mathfrak{S}^{\prime}$ an a-preserving bounded $\mathcal{H}$-morphism. Then, for each formula $A$ of $L_{\square \diamond}^{\mathcal{H}}$ and state $\mathfrak{s}$ of $\mathfrak{M}$,

$$
a \cdot v(\mathfrak{s}, A)=a \cdot v^{\prime}(f(\mathfrak{s}), A)
$$

Proof. The proof runs again by induction on A. We omit the routine cases of the propositional connectives and proceed to the modal operators. We will provide (half of) the argument for $\square$, as an indication of the techniques employed. We split our proof into two parts:

$($ Part 1, $\geq) a \cdot v\left(\mathfrak{s}, \square A_{1}\right) \geq a \cdot v^{\prime}\left(f(\mathfrak{s}), \square A_{1}\right)$ : 


$$
\begin{array}{rlrl}
a \cdot v\left(\mathfrak{s}, \square A_{1}\right) & =a \cdot \prod_{\mathfrak{t} \in \mathfrak{S}}\left(\mathfrak{g}(\mathfrak{s}, \mathfrak{t}) \Rightarrow v\left(\mathfrak{t}, A_{1}\right)\right) & \\
& =\prod_{\mathfrak{t} \in \mathfrak{S}}\left(a \cdot\left(\mathfrak{g}(\mathfrak{s}, \mathfrak{t}) \Rightarrow v\left(\mathfrak{t}, A_{1}\right)\right)\right) & & \left(\text { by } \mathcal{H}_{10}\right) \\
& =\prod_{\mathfrak{t} \in \mathfrak{S}} a \cdot\left(a \cdot \mathfrak{g}(\mathfrak{s}, \mathfrak{t}) \Rightarrow a \cdot v\left(\mathfrak{t}, A_{1}\right)\right) & \\
& \left.\geq \prod_{\mathfrak{t} \in \mathfrak{S}} a \cdot(\underbrace{a \cdot \mathfrak{g}^{\prime}(f(\mathfrak{s}), f(\mathfrak{t}))}_{\text {forth }} \Rightarrow \underbrace{a \cdot v^{\prime}\left(f(\mathfrak{t}), A_{1}\right.}_{\text {Induct. Hypothesis }})\right) \quad\left(\mathcal{H}_{4},\right. \text { Def. 4.8) } \\
& =\prod_{\mathfrak{t} \in \mathfrak{S}} a \cdot\left(\mathfrak{g}^{\prime}(f(\mathfrak{s}), f(\mathfrak{t})) \Rightarrow v^{\prime}\left(f(\mathfrak{t}), A_{1}\right)\right) & \\
& =a \cdot \prod_{\mathfrak{t} \in \mathfrak{S}}\left(\mathfrak{g}^{\prime}(f(\mathfrak{s}), f(\mathfrak{t})) \Rightarrow v^{\prime}\left(f(\mathfrak{t}), A_{1}\right)\right) & \\
& \geq a \cdot \prod_{\mathfrak{t}^{\prime} \in \mathfrak{S}_{10}}\left(\mathfrak{g}^{\prime}\left(f(\mathfrak{s}), \mathfrak{t}^{\prime}\right) \Rightarrow v^{\prime}\left(\mathfrak{t}^{\prime}, A_{1}\right)\right) & \left(f[\mathfrak{S}] \subseteq \mathfrak{S}^{\prime}\right) \\
& =a \cdot v^{\prime}\left(f(\mathfrak{s}), \square A_{1}\right) & (\text { Def. 3.1) }
\end{array}
$$

(Part 2, $\leq) a \cdot v^{\prime}\left(f(\mathfrak{s}), \square A_{1}\right) \geq a \cdot v\left(\mathfrak{s}, \square A_{1}\right)$. This part requires an application of the Axiom of Choice, along with the back condition, to ensure that a suitable $h: \mathfrak{S}^{\prime} \rightarrow \mathfrak{S}$ exists, which, for every $\mathfrak{t}^{\prime} \in \mathfrak{S}^{\prime}$ (such that $\left.a \cdot \mathfrak{g}^{\prime}\left(f(\mathfrak{s}), \mathfrak{t}^{\prime}\right) \neq \perp\right)$, picks one state $h\left(\mathfrak{t}^{\prime}\right) \in$ $f^{-1}\left[\left\{\mathfrak{t}^{\prime}\right\}\right] \subseteq \mathfrak{S}$, such that $a \cdot \mathfrak{g}^{\prime}\left(f(\mathfrak{s}), \mathfrak{t}^{\prime}\right)=a \cdot \mathfrak{g}\left(\mathfrak{s}, h\left(\mathfrak{t}^{\prime}\right)\right)$. Details are left to the reader.

The result at the level of frames follows immediately and is stated without proof.

Theorem 4.10 Let $\mathfrak{F}=\langle\mathfrak{S}, \mathfrak{g}\rangle$ and $\mathfrak{F}^{\prime}=\left\langle\mathfrak{S}^{\prime}, \mathfrak{g}^{\prime}\right\rangle$ be two $\mathcal{H}$-frames, and $f$ a surjective a-bounded morphism $: \mathfrak{F} \rightarrow^{a} \mathfrak{F}^{\prime}$. Then, for every $X \in L_{\square \diamond}^{\mathcal{H}}, \mathfrak{F} \Vdash_{a} X$ implies $\mathfrak{F}^{\prime} \Vdash_{a} X$.

Example 4.11 It is tempting to consider a many-valued analog of the reflexive frames: the $\mathcal{H}$-frames, in which every label $\mathfrak{g}(\mathfrak{s}, \mathfrak{s})$ has the value $a$, where $a \neq \perp$ and $a \neq \top$. Assume that there exists a formula $X \in L_{\square \diamond}^{\mathcal{H}}$ which defines this class of $\mathcal{H}$-frames. Consider the $\mathcal{H}$-frame $\mathfrak{F}_{1}=\left\langle\mathbb{N}, \mathfrak{g}_{1}\right\rangle$, where for every $n \in \mathbb{N}, \mathfrak{g}_{1}(n, n)=a, \mathfrak{g}_{1}(n, n+1)=$ $\top$, and $\mathfrak{g}_{1}(m, n)=\perp$, for every other pair $m, n$. Then $\mathfrak{F}_{1} \Vdash X$. Now, consider the $\mathcal{H}$ frame $\mathfrak{F}_{2}=\left\langle\{0\}, \mathfrak{g}_{2}\right\rangle$, where $\mathfrak{g}_{2}(0,0)=\top$. The function $f: \mathfrak{F}_{1} \rightarrow \mathfrak{F}_{2}$ with $f(n)=0$ for every $n \in \mathbb{N}$, is a surjective $T$-preserving bounded morphism (check with the back and forth conditions). However, $\mathfrak{F}_{2} \not \forall X$, a contradiction. Further on this example, it has been proved in [15, Theorem 16(1)] that the class of $\mathcal{H}$-frames satisfying the condition $\forall \mathfrak{s}(\mathfrak{g}(\mathfrak{s}, \mathfrak{s}) \geq a)$ is modally definable by the formula $\mathbf{T}^{a}: \square A \supset(a \supset A)$. It follows that the property of $\mathcal{H}$-frames $\forall \mathfrak{s}(\mathfrak{g}(\mathfrak{s}, \mathfrak{s}) \leq a)$ is not definable, lest the generalization of reflexivity in the beginning of the example be.

The following theorem asserts that, a well-known intimate relation between the notions of generated subframes and bounded morphisms [10, Lemma 3.2.2], carries through in the many-valued setting. 
Proposition 4.12 If $f$ is an a-preserving bounded $\mathcal{H}$-morphism from $\mathfrak{M}_{0}=\left\langle\mathfrak{S}_{0}, \mathfrak{g}_{0}, v_{0}\right\rangle$ to $\mathfrak{M}=\langle\mathfrak{S}, \mathfrak{g}, v\rangle$ (from $\mathfrak{F}_{0}=\left\langle\mathfrak{S}_{0}, \mathfrak{g}_{0}\right\rangle$ to $\mathfrak{F}=\langle\mathfrak{S}, \mathfrak{g}\rangle$ ), then the image of $\mathfrak{M}_{0}\left(\mathfrak{F}_{0}\right)$ under $f$ is an a-preserving generated submodel (subframe) of $\mathfrak{M}(\mathfrak{F})$.

\subsection{Canonical Extensions of $\mathcal{H}$-frames and $\mathcal{H}$-models}

The construction of canonical extensions of relational frames is very interesting in its own right as it imports elements of classical model theory in modal logic. Canonical extensions are also called ultrafilter extensions as the set of states in the resulting frame consists of the ultrafilters over the set of states in the initial frame. This construction has been employed in many aspects of classical modal logic: as a tool for building modally saturated models [2, Chapter 2.5], as a fundamental tool in definability theory (cf. the Goldblatt-Thomason Theorem [13]) and in the metamathematical investigations of modality in the '70s and ' 80 s (see [22] for an example and $[2,12]$ for references).

Setting the stage. The process of building the canonical extension $\left(\mathcal{F}^{+}\right)_{+}$of a relational frame $\mathcal{F}$ consists classically of two steps: (i) building its full complex algebra $\mathcal{F}^{+}$, the prime example of a Boolean Algebra with Operators (BAO), and (ii) constructing the ultrafilter frame $\left(\mathcal{F}^{+}\right)_{+}$of the full complex algebra (see [2, Ch. 5] and [11]). We are going to provide the many-valued analogs of these steps, starting with the analog of a full complex algebra. To this end, we employ the direct power $\mathcal{H}^{\mathfrak{G}}$ of $\mathcal{H}$. In doing so, we imitate the classical construction, generalizing the fact that every powerset algebra is isomorphic to a direct power of $\mathbf{2}$. To assist in seeing the analogy with the classical case, let us remind that every subset of the set of states $\mathfrak{S}$ can be identified with its characteristic function, which is a function from $\mathfrak{S}$ to $\mathbf{2}$. For the basic connection between logic and universal algebra, comprising the elementary facts we generalize in this Section, the reader can consult the excellent notes of Y. Venema [23].

Definition 4.13 Let $\mathfrak{F}=\langle\mathfrak{S}, \mathfrak{g}\rangle$ be an $\mathcal{H}$-frame for $L_{\square \triangleright}^{\mathcal{H}}$. We augment the power algebra $\mathcal{H}^{\mathfrak{S}}$ with two operators $\mathrm{m}_{\mathfrak{g}}, \mathrm{l}_{\mathfrak{g}}$, to obtain a a Heyting Algebra with Operators, denoted as

$$
\mathrm{F}=\left\langle H^{\mathfrak{S}},+_{\mathrm{F}}, \cdot_{\mathrm{F}}, \Rightarrow_{\mathrm{F}}, \perp_{\mathrm{F}}, \mathrm{m}_{\mathfrak{g}}, 1_{\mathfrak{g}}\right\rangle
$$

where the two operators $\mathrm{m}_{\mathfrak{g}}$ and $\mathrm{l}_{\mathfrak{g}}: \mathcal{H}^{\mathfrak{S}} \rightarrow \mathcal{H}^{\mathfrak{S}}$ are coordinate-wise defined as follows: for every $\mathfrak{s} \in \mathfrak{S}$ and $\mathfrak{f} \in \mathcal{H}^{\mathfrak{S}}$,

$$
\mathrm{m}_{\mathfrak{g}}(\mathfrak{f})(\mathfrak{s})=\sum_{\mathfrak{t} \in \mathfrak{S}}(\mathfrak{g}(\mathfrak{s}, \mathfrak{t}) \cdot \mathfrak{f}(\mathfrak{t})) \quad \mathrm{l}_{\mathfrak{g}}(\mathfrak{f})(\mathfrak{s})=\prod_{\mathfrak{t} \in \mathfrak{S}}(\mathfrak{g}(\mathfrak{s}, \mathfrak{t}) \Rightarrow \mathfrak{f}(\mathfrak{t}))
$$

The operators $m_{\mathfrak{g}}$ and $l_{\mathfrak{g}}$ will interpret $\diamond$ and $\square$ respectively and it can be easily checked that in the case of the classical language $L_{\square \diamond}^{2}$, they fall respectively to $\mathrm{m}_{\mathcal{R}}(X)$ (the states that can 'see' a state in $X$ ) and $\mathrm{l}_{\mathcal{R}}(X)$ (the states that 'see' only states in $X$ ). For an element $t \in \mathcal{H}$, we denote by $[t]$ the constant function (an element of $\mathcal{H}^{\mathfrak{S}}$ ) which maps every state $\mathfrak{s} \in \mathfrak{S}$ to $t$. 
Remark 4.14 (Proviso \& Notation) In this section, the algebra $\mathcal{H}$ underlying $L_{\square \diamond}^{\mathcal{H}}$ is finite. An algebraic operation without a subscript refers to $\mathcal{H}$ : for instance, $\Rightarrow$ means $\Rightarrow_{\mathcal{H}}$ while $\Rightarrow_{\mathrm{F}}$ is explicitly denoted. In the interests of readability, we are going however to deviate from the standard notation $\left(\mathcal{F}^{+}\right)_{+}$and use fonts to differentiate between the different constructions: we move from an $\mathcal{H}$-frame $\mathfrak{F}$ to its associated Heyting algebra with operators $\mathrm{F}$ and then to its canonical extension $\mathfrak{F}^{\mathfrak{E}}$.

The following lemma gathers some useful properties of the operators $m_{\mathfrak{g}}$ and $\mathrm{l}_{\mathfrak{g}}$. The third one is not actually used in the proofs to follow, but we record it here for completeness. The details of the proofs are left to the reader.

Lemma 4.15 For every $a \in \mathcal{H}$ and $\mathrm{f}_{1}, \mathrm{f}_{2} \in \mathrm{F}$,

1. $\mathrm{f}_{1} \leq_{\mathrm{F}} \mathrm{f}_{2}$ implies $\mathrm{l}_{\mathfrak{g}}\left(\mathrm{f}_{1}\right) \leq_{\mathrm{F}} \mathrm{l}_{\mathfrak{g}}\left(\mathrm{f}_{2}\right) \quad$ (monotonicity of $\mathrm{l}_{\mathfrak{g}}$ )

2. $l_{\mathfrak{g}}\left(f_{1} \cdot f_{F}\right)=l_{\mathfrak{g}}\left(f_{1}\right) \bullet_{F} l_{\mathfrak{g}}\left(f_{2}\right) \quad$ (multiplicativity of $l_{\mathfrak{g}}$ )

3. $\mathrm{m}_{\mathfrak{g}}\left(\mathrm{f}_{1}+_{\mathrm{F}} \mathrm{f}_{2}\right)=\mathrm{m}_{\mathfrak{g}}\left(\mathrm{f}_{1}\right)+_{\mathrm{F}} \mathrm{m}_{\mathfrak{g}}\left(\mathrm{f}_{2}\right) \quad$ (additivity of $\mathrm{m}_{\mathfrak{g}}$ )

4. $1_{\mathfrak{g}}\left([a] \Rightarrow_{\mathrm{F}} \mathrm{f}\right)=\left([a] \Rightarrow_{\mathrm{F}} \mathrm{l}_{\mathfrak{g}}(\mathrm{f})\right)$

5. $\mathrm{l}_{\mathfrak{g}}\left(\mathrm{f} \Rightarrow_{\mathrm{F}}[a]\right)=\left(\mathrm{m}_{\mathfrak{g}}(\mathrm{f}) \Rightarrow_{\mathrm{F}}[a]\right)$

The following definition generalizes the classical function $V(\varphi)$ of a relational model, which maps a formula $\varphi$ to the set of states in which it is true.

Definition 4.16 Given an $\mathcal{H}$-model $\mathfrak{M}=\langle\mathfrak{S}, \mathfrak{g}, v\rangle$ and a formula $\varphi \in L_{\square \diamond}^{\mathcal{H}}(\Phi)$, we define $[\varphi]_{\mathfrak{M}}: L_{\square \diamond}^{\mathcal{H}}(\Phi) \rightarrow \mathrm{F}$ to be the function of $\mathcal{H}^{\mathfrak{S}}$ defined by: for every $\mathfrak{s} \in \mathfrak{S}$, $[\varphi]_{\mathfrak{M}}(\mathfrak{s})=v(\mathfrak{s}, \varphi)$

Of course, for $t \in \mathcal{H},[t]_{\mathfrak{M}}$ is $[t]$. Whenever it is implied from the context, the subscript in $[\varphi]_{\mathfrak{M}}$ will be omitted. It can be now easily verified that this function correctly captures the interplay between the modal operators and their algebraic incarnations.

Fact $4.17[\diamond \varphi]_{\mathfrak{M}}=\mathrm{m}_{\mathfrak{g}}\left([\varphi]_{\mathfrak{M}}\right)$ and $[\square \varphi]_{\mathfrak{M}}=1_{\mathfrak{g}}\left([\varphi]_{\mathfrak{M}}\right)$.

Proof. For an arbitrary state $\mathfrak{s} \in \mathfrak{S}$, it holds that

$[\diamond \varphi]_{\mathfrak{M}}(\mathfrak{s})=v(\mathfrak{s}, \diamond \varphi) \stackrel{\text { Def. }}{=}{ }^{3.1} \sum_{\mathfrak{t} \in \mathfrak{S}} \mathfrak{g}(\mathfrak{s}, \mathfrak{t}) \cdot v(\mathfrak{t}, \varphi) \stackrel{\text { Def. }}{=}{ }^{4.16} \sum_{\mathfrak{t} \in \mathfrak{S}} \mathfrak{g}(\mathfrak{s}, \mathfrak{t}) \cdot[\varphi]_{\mathfrak{M}}(\mathfrak{t}) \stackrel{\text { Def. }}{=}{ }^{.13} \mathrm{~m}_{\mathfrak{g}}\left([\varphi]_{\mathfrak{M}}\right)(\mathfrak{s})$

The proof for $[\square \varphi]_{\mathfrak{M}}$ is similar. 
In order to define the canonical extension of an $\mathcal{H}$-frame, we have to define first its set of states, the many-valued analog of an ultrafilter over $\mathfrak{S}$. Before doing so, we remind the reader that there exists an one-to-one correspondence between the set of ultrafilters of any Boolean algebra B and the set of homomorphisms of B onto $\mathbf{2}$ [23, 1 ]. Every powerset boolean algebra is identified with a direct power of $\mathbf{2}$ and this grasps the intuition behind our choice to define the states of the canonical extension in the general many-valued case, using the homomorphisms from the Heyting algebra $\mathrm{F}$ onto $\mathcal{H}$, but not all of them. We will use those that 'properly' interpret the elements of $\mathcal{H}$.

Definition 4.18 Let $\mathrm{F}$ be the algebra arising from an $\mathcal{H}$-frame $\mathfrak{F}$. A homomorphism $\mathbf{h}$ from $\mathrm{F}$ to $\mathcal{H}$, which maps $[t]$ to $t$, for every $t \in \mathcal{H}$, is called $\mathcal{H}$-proper ${ }^{4}$.

Observe that an $\mathcal{H}$-proper homomorphism is trivially onto. The following definition provides the algebraic equivalent of the logical notion of consistency. In the manyvalued case this notion is more refined, cf. the discussion in [7, Sect. 4]. Moreover, the equivalent of the well-known notion of sets with the finite intersection property $[4, \mathrm{Ch}$. $4.1]$ is also more complex.

Definition 4.19 Let $T$ and $f$ be respectively a subset and an element of $F$. An $\mathcal{H}$ proper homomorphism $\mathbf{h}$ is $\mathbf{f}$-compatible w.r.t. $\mathrm{T}$ iff for every finite subset $\mathrm{T}_{0}$ of $\mathrm{T}$,

$$
\prod_{g \in T_{0}} \mathbf{h}(g) \not \mathbf{h}(f) .
$$

Let $S, T$ and $f$ be respectively two subsets and an element of $F$. We say that the pair $\langle\mathrm{S}, \mathrm{T}\rangle$ has the finite meet property with respect to $\mathrm{f}$ (short: has the f-f.m.p.) iff for every pair of finite subsets $\mathrm{S}_{0} \subseteq \mathrm{S}$ and $\mathrm{T}_{0} \subseteq \mathrm{T}$, there exists an $\mathcal{H}$-proper homomorphism $\mathbf{h}$ such that:

- $\mathbf{h}\left[S_{0}\right]=\{\top\}$

- $\prod_{\mathrm{g} \in \mathrm{T}_{0}} \mathbf{h}(\mathrm{g}) \not \mathbf{L}(\mathrm{f})$

We say that $\left\langle S_{m}, T\right\rangle$ is maximal ${ }^{5}$ with respect to the f-f.m.p., iff $\left\langle S_{m}, T\right\rangle$ has the f-f.m.p., and for every proper extension $S^{\prime}$ of $S_{m}$ it cannot be the case that $\left\langle S^{\prime}, T\right\rangle$ has the f-f.m.p..

\footnotetext{
${ }^{4}$ If $\mathcal{H}$ is to be considered as part of the signature, every homomorphism is $\mathcal{H}$-proper and this definition does not make sense. From the perspective of logic this is not necessary as the elements of $\mathcal{H}$ are 'special' atomic formulae, hence the definition of $\mathcal{H}$-proper homomorphisms.

${ }^{5}$ As in Fitting's canonical model argument in [7], we have been unable to avoid the pair $\langle\mathrm{S}, \mathrm{T}\rangle$ and work only with its second component. In $[7,17]$ the sets of sentences with the proof-theoretic equivalent of this maximality property are called maximal left-consistent sets, as it is the first component which is maximized. We do not wish to complicate further the terminology in this paper: whenever we refer to a maximal pair $\left\langle S_{m}, T\right\rangle$ we always mean that its first component $S_{m}$ is maximal with respect to the f-f.m.p.; hence the subscript to $S_{m}$.
} 
Note that if $\left\langle S_{m}, T\right\rangle$ is maximal with respect to the f-f.m.p., then $[T]=T_{F}$ is in $S_{m}$. We argue now about the existence of maximal sets with the f-f.m.p., and their essential property, that will allow us to build $\mathcal{H}$-proper homomorphisms, f-compatible w.r.t. $\mathrm{T}$, out of them. We will first state, without proof, a useful lemma. In the results to follow, we assume that $\mathrm{F}$ is the algebra arising from an $\mathcal{H}$-frame $\mathfrak{F}$.

Lemma 4.20 If a pair $\left\langle\mathrm{S}_{\mathrm{m}}, \mathrm{T}\right\rangle$ of subsets of $\mathrm{F}$ is maximal with respect to the $\mathrm{f}-f . m . p$., $\mathrm{S}_{\mathrm{m}}$ is a filter in (the lattice reduct of) $\mathrm{F}$.

Proposition 4.21 If $\langle\mathrm{S}, \mathrm{T}\rangle$ has the f-f.m.p., then it can be extended to a maximal $\left\langle\mathrm{S}_{\mathrm{m}}, \mathrm{T}\right\rangle$ with the $\mathrm{f}-f . m . p$.. Moreover, it holds that for every $\mathrm{f} \in \mathrm{F}$, there exists a unique $t \in \mathcal{H}$ such that $\left(\mathrm{f} \Leftrightarrow_{\mathrm{F}}[t]\right) \in \mathrm{S}_{\mathrm{m}}$.

Proof. The existence of $\mathrm{S}_{\mathrm{m}}$ is a typical application of Zorn's lemma. For the existence claim,the finiteness of $\mathcal{H}=\left\{t_{1}, \ldots, t_{n}\right\}$ is essential. For the uniqueness claim, assume that there exist two different members $t_{1}, t_{2} \in \mathcal{H}$ and a $\mathrm{g}^{\prime} \in \mathrm{F}$ such that both $\mathrm{g}^{\prime} \Leftrightarrow_{\mathrm{F}}\left[t_{1}\right]$ and $\mathrm{g}^{\prime} \Leftrightarrow_{\mathrm{F}}\left[t_{2}\right]$ are in $\mathrm{S}_{\mathrm{m}}$. Lemma 4.20 asserts that $\mathrm{S}_{\mathrm{m}}$ is upwards closed and closed under meet, which implies that $\left[t_{1}\right] \Leftrightarrow_{\mathrm{F}}\left[t_{2}\right]$ should be in $\mathrm{S}_{\mathrm{m}}$. But for any $\mathcal{H}$-proper function $\mathbf{h}$, $\mathbf{h}\left(\left[t_{1}\right] \Leftrightarrow\left[t_{2}\right]\right) \neq \top$, since $t_{1} \neq t_{2}$, and hence trivially, $\left\langle\mathrm{S}_{\mathrm{m}}, \mathbf{T}\right\rangle$ would have not have the f-f.m.p.

We can now prove that we can obtain suitable $\mathcal{H}$-proper functions from a maximal pair $\left\langle\mathrm{S}_{\mathrm{m}}, \mathrm{T}\right\rangle$. It is actually the first part of Theorem 4.23 that we will use; the other part is recorded in the interests of completeness. Before going to the theorem, we have to state a useful Proposition, which is proved by applying simple properties of congruence relation in Heyting algebras to filter $S_{m}$ (Lemma 4.20).

Proposition 4.22 For any $\star \in\{\cdot,+, \Rightarrow\}$ and (its corresponding) $\star_{\mathrm{F}} \in\left\{\cdot_{\mathrm{F}},+_{\mathrm{F}}, \Rightarrow_{\mathrm{F}}\right\}$, if $\left\{\left(\mathrm{g}_{1} \Leftrightarrow_{\mathrm{F}}\left[t_{1}\right]\right),\left(\mathrm{g}_{2} \Leftrightarrow_{\mathrm{F}}\left[t_{2}\right]\right)\right\} \subseteq \mathrm{S}_{\mathrm{m}}$, then $\left(\mathrm{g}_{1} \star_{\mathrm{F}} \mathrm{g}_{2} \Leftrightarrow_{\mathrm{F}}\left[t_{1} \star t_{2}\right]\right) \in \mathrm{S}_{\mathrm{m}}$.

Theorem 4.23 If $\left\langle\mathrm{S}_{\mathrm{m}}, \mathrm{T}\right\rangle$ is maximal with respect to the $\mathrm{f}-f . m . p$. , then the function $\mathbf{h}_{\mathrm{s}_{\mathrm{m}}}: \mathrm{F} \rightarrow \mathcal{H}$ defined by:

$$
\text { for every } \mathrm{g} \in \mathrm{F}, \quad \mathbf{h}_{\mathrm{S}_{\mathrm{m}}}(\mathrm{g})=\text { the unique } t \in \mathcal{H} \text { s.t. }\left(\mathrm{g} \Leftrightarrow_{\mathrm{F}}[t]\right) \in \mathrm{S}_{\mathrm{m}} \text {, }
$$

is $\mathrm{f}$-compatible w.r.t. $\mathrm{T}$.

Conversely, if $\mathbf{h}$ is $\mathbf{f}$-compatible w.r.t. a set $\mathrm{T} \subseteq \mathrm{F}$, then there exists a (unique) set $\mathrm{S}_{\mathrm{m}}$ such that $\left\langle\mathrm{S}_{\mathrm{m}}, \mathrm{T}\right\rangle$ is maximal with respect to the $\mathrm{f}-f . m . p$.

Proof. We have to show first that $\mathbf{h}_{\mathrm{s}_{\mathrm{m}}}$ is $\mathcal{H}$-proper . To this end, let $\star \in\{\cdot,+, \Rightarrow\}$ and $\star_{\mathrm{F}} \in\left\{\cdot_{\mathrm{F}},+_{\mathrm{F}}, \Rightarrow_{\mathrm{F}}\right\}$. Then, for every $\mathrm{g}_{1}$ and $\mathrm{g}_{2}$ in $\mathrm{F}$, there exist unique $t_{1}$ and $t_{2}$ in $\mathcal{H}$, s.t. $\left\{\left(\mathrm{g}_{1} \Leftrightarrow_{\mathrm{F}}\left[t_{1}\right]\right),\left(\mathrm{g}_{2} \Leftrightarrow_{\mathrm{F}}\left[t_{2}\right]\right)\right\} \subseteq \mathrm{S}_{\mathrm{m}}$. By Proposition 4.22: $\left(\mathrm{g}_{1} \star_{\mathrm{F}} \mathrm{g}_{2} \Leftrightarrow_{\mathrm{F}}\left[t_{1} \star t_{2}\right]\right) \in \mathrm{S}_{\mathrm{m}}$, and hence, $\mathbf{h}_{\mathrm{S}_{\mathrm{m}}}\left(\mathrm{g}_{1} \star_{\mathrm{F}} \mathrm{g}_{2}\right)=t_{1} \star t_{2}=\mathbf{h}_{\mathrm{S}_{\mathrm{m}}}\left(\mathrm{g}_{1}\right) \star \mathbf{h}_{\mathrm{S}_{\mathrm{m}}}\left(\mathrm{g}_{2}\right)$. It follows that $\mathbf{h}_{\mathrm{S}_{\mathrm{m}}}$ is a homomorphism from $\mathrm{F}$ to $\mathcal{H}$. Also, for every $t \in \mathcal{H}, \mathbf{h}([t])=t$, since $\left([t] \Leftrightarrow_{\mathrm{F}}[t]\right)=[\mathrm{T}]$ is in $\mathrm{S}_{\mathrm{m}}$. Thus, by Definition $4.18 \mathbf{h}_{\mathrm{s}_{\mathrm{m}}}$ is an $\mathcal{H}$-proper function. 
Assume further, that $\mathbf{h}_{\mathrm{s}_{\mathrm{m}}}$ is not $\mathbf{f}$-compatible w.r.t. $\mathrm{T}$. Then, there exists a finite $\mathrm{T}_{0} \subseteq \mathrm{T}$, such that $\prod_{\mathrm{g} \in \mathrm{T}_{0}} \mathbf{h}_{\mathrm{s}_{\mathrm{m}}}(\mathrm{g}) \leq \mathbf{h}_{\mathrm{s}_{\mathrm{m}}}(\mathrm{f})$. Since $\mathbf{h}_{\mathrm{s}_{\mathrm{m}}}$ is a homomorphism, it holds that $\mathbf{h}_{\mathrm{S}_{\mathrm{m}}}\left(\left(\prod \mathrm{T}_{0}\right) \Rightarrow_{\mathrm{F}} \mathrm{f}\right)=\top$, or equivalently $\left(\left(\prod \mathrm{T}_{0}\right) \Rightarrow_{\mathrm{F}} \mathrm{f}\right) \in \mathrm{S}_{\mathrm{m}}$. Then, the pair $\left\langle\left\{\left(\prod T_{0}\right) \Rightarrow_{F} f\right\}, T_{0}\right\rangle$ witnesses that $\left\langle S_{m}, T\right\rangle$ does not have the f-f.m.p., since every $\mathcal{H}$ proper $\mathbf{h}$ such that $\mathbf{h}\left(\left\{\prod T_{0} \Rightarrow_{\mathrm{F}} f\right\}\right)=\{\top\}$ satisfies $\mathbf{h}\left(\prod \mathrm{T}_{0}\right) \leq \mathbf{h}(\mathbf{f})$; we have reached a contradiction.

For the second part of the theorem, if $\mathbf{h}$ is $\mathbf{f}$-compatible w.r.t. $\mathrm{T} \subseteq \mathrm{F}$, then the desired $\mathbf{S}_{\mathrm{m}}$ is the set $\mathbf{h}^{-1}[\{\top\}]=\{\mathrm{g} \in \mathrm{F}: \mathbf{h}(\mathrm{g})=\top\}$. Indeed,

- $\left\langle\mathrm{S}_{\mathrm{m}}, \mathrm{T}\right\rangle$ has the f-f.m.p.: for every pair of finite subsets $\mathrm{S}_{0} \subseteq \mathrm{S}_{\mathrm{m}}$ and $\mathrm{T}_{0} \subseteq \mathrm{T}$, $\mathbf{h}\left[\mathrm{S}_{0}\right]=\{\top\}$ by the definition of $\mathbf{S}_{\mathrm{m}}$ and $\prod_{\mathrm{g} \in \mathrm{T}_{0}} \mathbf{h}(\mathrm{g}) \not{L} \mathbf{h}(\mathrm{f})$ since $\mathbf{h}$ is $\mathbf{f}$-compatible w.r.t. T.

- $\left\langle\mathrm{S}_{\mathrm{m}}, \mathrm{T}\right\rangle$ is maximal w.r.t. the f-f.m.p.: extend $\left\langle\mathrm{S}_{\mathrm{m}}, \mathrm{T}\right\rangle$ to a maximal $\left\langle\mathrm{S}_{\mathrm{m}}{ }^{\prime}, \mathrm{T}\right\rangle$ with the f-f.m.p., according to Proposition 4.21. We will show that $S_{m}=S_{m}{ }^{\prime}$. Let $\mathrm{g} \in \mathrm{S}_{\mathrm{m}}{ }^{\prime}$. Then, by properties $\mathcal{H}_{2}$ and $\mathcal{H}_{3},\left(\mathrm{~g} \Leftrightarrow_{\mathrm{F}}[\mathrm{T}]\right) \in \mathrm{S}_{\mathrm{m}}{ }^{\prime}$. Now, since $\mathbf{h}$ is a function, there exists a $t \in \mathcal{H}$ s.t. $\mathbf{h}(\mathrm{g})=t$. Hence, $\mathbf{h}\left(\mathrm{g} \Leftrightarrow_{\mathrm{F}}[t]\right)=\top$, which implies $\left(\mathrm{g} \Leftrightarrow_{\mathrm{F}}[t]\right) \in \mathrm{S}_{\mathrm{m}} \subseteq \mathrm{S}_{\mathrm{m}}{ }^{\prime}$, and this forces $t$ be $T$. That is, $\mathbf{h}(\mathrm{g})=\top$ and hence $\mathrm{g} \in \mathrm{S}_{\mathrm{m}}$.

The analog of principal ultrafilters. The principal ultrafilters of a powerset algebra are generated by the singleton sets and they play an important role in the classical ultrafilter extensions of frames. The following definition properly generalizes this notion in the context of $\mathcal{H}$-frames and their corresponding Heyting algebras with operators.

Definition 4.24 Assume $\mathfrak{F}=\langle\mathfrak{S}, \mathfrak{g}\rangle$ is an $\mathcal{H}$-frame and $\mathrm{F}$ is its corresponding Heyting algebra with operators. For every $\mathfrak{s} \in \mathfrak{S}$, the point-generated function associated to $\mathfrak{s}$ is the function $\pi_{\mathfrak{s}}: \mathrm{F} \rightarrow \mathcal{H}$, defined by: for every $\mathrm{f} \in \mathrm{F}, \pi_{\mathfrak{s}}(\mathrm{f})=\mathrm{f}(\mathfrak{s})$

The following proposition verifies that point-generated functions are of interest for the purposes of this section. Its proof is based on the coordinate-wise definition of operations in the direct power of $\mathcal{H}$, on which $\mathrm{F}$ is based.

Proposition $4.25 \pi_{\mathfrak{s}}$ is an $\mathcal{H}$-proper function.

A first use of point-generated functions is in the following lemma, which will be needed in the proof of our main result in this section.

Lemma 4.26 If $\mathrm{T}$ is a subset of $\mathrm{F}$ such that for every finite $\mathrm{T}_{0} \subseteq \mathrm{T}, \prod \mathrm{T}_{0} \mathbb{L}_{\mathrm{F}} \mathrm{f}$, then $\langle\emptyset, \mathrm{T}\rangle$ has the f-f.m.p. 
Proof. To prove that $\langle\emptyset, T\rangle$ has the f-f.m.p., it suffices to show that for every finite $\mathrm{T}_{0} \subseteq \mathrm{T}$, there exists an $\mathcal{H}$-proper h s.t. $\prod_{\mathrm{g} \in \mathrm{T}_{0}} \mathbf{h}(\mathrm{g}) \not \underline{\mathbf{h}}(\mathrm{f})$. Since $\prod \mathrm{T}_{0} \not \underline{Z}_{\mathrm{F}} \mathrm{f}$, or, equivalently, $\left(\left(\prod \mathrm{T}_{0}\right) \Rightarrow_{\mathrm{F}} \mathrm{f}\right) \neq \mathrm{T}_{\mathrm{F}}\left(\right.$ by $\left.\mathcal{H}_{2}\right)$, there exists an $\mathfrak{s} \in \mathfrak{S}$ such that $\left(\left(\prod \mathrm{T}_{0}\right) \Rightarrow_{\mathrm{F}}\right.$ f) $(\mathfrak{s}) \neq \top$. Then, the point-generated $\mathcal{H}$-proper function $\pi_{\mathfrak{s}}$ is the required function, since $\pi_{\mathfrak{s}}\left(\left(\prod \mathrm{T}_{0}\right) \Rightarrow_{\mathrm{F}} \mathrm{f}\right)=\left(\left(\prod \mathrm{T}_{0}\right) \Rightarrow_{\mathrm{F}} \mathrm{f}\right)(\mathfrak{s}) \neq \top$, and thus $\prod_{\mathrm{g} \in \mathrm{T}_{0}} \pi_{\mathfrak{s}}(\mathrm{g}) \not \leq \pi_{\mathfrak{s}}(\mathrm{f})$.

Canonical extensions and the main theorem. We are now ready to define the canonical extension of an $\mathcal{H}$-frame and an $\mathcal{H}$-model. The set of states is the set of all $\mathcal{H}$-proper functions. The accessibility function defined below collapses to the original definition of the accessibility relation between ultrafilters (see [2, Ch. 2]) in the case of the language $L_{\square \diamond}^{2}$, as the reader can verify.

Definition 4.27 Let $\mathfrak{F}=\langle\mathfrak{S}, \mathfrak{g}\rangle$ be an $\mathcal{H}$-frame. Its canonical extension is the frame $\mathfrak{F}^{\mathfrak{E}}=\left\langle\mathfrak{S}^{\mathfrak{E}}, \mathfrak{g}^{\mathfrak{E}}\right\rangle$, where $\mathfrak{S}^{\mathfrak{E}}$ is the set of $\mathcal{H}$-proper homomorphisms from $\mathrm{F}$ to $\mathcal{H}$ and the accessibility function between two states $\left(\mathcal{H}\right.$-proper functions) $\mathbf{h}_{1}$ and $\mathbf{h}_{2}$ is defined by

$$
\mathfrak{g}^{\mathfrak{E}}\left(\mathbf{h}_{1}, \mathbf{h}_{2}\right)=\prod_{\mathbf{f} \in \mathrm{F}}\left(\mathbf{h}_{2}(\mathbf{f}) \Rightarrow \mathbf{h}_{1}\left(\mathrm{~m}_{\mathfrak{g}}(\mathfrak{f})\right)\right) \cdot \prod_{\mathfrak{f} \in \mathrm{F}}\left(\mathbf{h}_{1}\left(\mathrm{l}_{\mathfrak{g}}(\mathbf{f})\right) \Rightarrow \mathbf{h}_{2}(\mathbf{f})\right)
$$

Further on, if $\mathfrak{M}=\langle\mathfrak{S}, \mathfrak{g}, v\rangle$ is an $\mathcal{H}$-model built on $\mathfrak{F}$, its canonical extension is the $\mathcal{H}$-model $\mathfrak{M}^{\mathfrak{E}}=\left\langle\mathfrak{S}^{\mathfrak{E}}, \mathfrak{g}^{\mathfrak{E}}, v^{\mathbb{E}}\right\rangle$, where the valuation function $v^{\mathfrak{E}}$ is defined by: for every $P \in \Phi, v^{\mathfrak{E}}(\mathbf{h}, P)=\mathbf{h}\left([P]_{\mathfrak{M}}\right)$.

Note that the definition of $v^{\mathbb{E}}$ is legitimate as it satisfies the requirement that, for every $t \in \mathcal{H}, v^{\mathfrak{E}}(\mathbf{h}, t)=\mathbf{h}\left([t]_{\mathfrak{M}}\right)=\mathbf{h}([t])=t$ (by Def. 3.1 and Def. 4.18). The main theorem for canonical extensions, stated below, verifies that the valuation function $v^{\mathfrak{E}}$ given above for propositional variables, can be lifted to every formula in $L_{\square \diamond}^{\mathcal{H}}(\Phi)$.

Theorem 4.28 For every formula $\varphi \in L_{\square \diamond}^{\mathcal{H}}(\Phi)$ and every $\mathcal{H}$-proper function $\mathbf{h} \in \mathfrak{S}^{\mathfrak{E}}$

$$
v^{\mathfrak{E}}(\mathbf{h}, \varphi)=\mathbf{h}\left([\varphi]_{\mathfrak{M}}\right) .
$$

Proof. The proof is by induction on $\varphi$. The propositional cases are fairly easy and are omitted. The cases of the modal operators are more complicated. We provide the proof for the case of $\varphi=\square \varphi^{\prime}$, and omit the similar proof for $\diamond$. The interested reader can check that our argument closely follows the relevant theorem of M. Fitting in his original paper [7, Theorem 7.6].

( $\square$, Part 1.) $\mathbf{h}\left(\left[\square \varphi^{\prime}\right]_{\mathfrak{M}}\right) \leq v^{\mathfrak{E}}\left(\mathbf{h}, \square \varphi^{\prime}\right)$ 


$$
\begin{aligned}
& v^{\mathfrak{E}}\left(\mathbf{h}, \square \varphi^{\prime}\right)=\prod_{\mathbf{h}^{\prime} \in \mathfrak{S}^{\mathfrak{E}}}\left(\mathfrak{g}^{\mathfrak{E}}\left(\mathbf{h}, \mathbf{h}^{\prime}\right) \Rightarrow v^{\mathfrak{E}}\left(\mathbf{h}^{\prime}, \varphi^{\prime}\right)\right) \\
& =\prod_{\mathbf{h}^{\prime} \in \mathfrak{S}^{\mathfrak{E}}}\left(\mathfrak{g}^{\mathfrak{E}}\left(\mathbf{h}, \mathbf{h}^{\prime}\right) \Rightarrow \mathbf{h}^{\prime}\left(\left[\varphi^{\prime}\right]_{\mathfrak{M}}\right)\right) \quad \text { (by Ind. Hyp.) } \\
& =\prod_{\mathbf{h}^{\prime} \in \mathfrak{S}^{\mathfrak{E}}}(\underbrace{\prod_{\mathbf{f} \in \mathbf{F}}\left(\mathbf{h}^{\prime}(\mathbf{f}) \Rightarrow \mathbf{h}\left(\mathrm{m}_{\mathfrak{g}}(\mathbf{f})\right)\right) \cdot \prod_{\mathbf{f} \in \mathbf{F}}\left(\mathbf{h}\left(l_{\mathfrak{g}}(\mathbf{f})\right) \Rightarrow \mathbf{h}^{\prime}(\mathbf{f})\right)}_{\text {Definition } 4.27} \Rightarrow \mathbf{h}^{\prime}\left(\left[\varphi^{\prime}\right]_{\mathfrak{M}}\right)) \\
& \geq \prod_{\mathbf{h}^{\prime} \in \mathfrak{S}^{\mathfrak{E}}}\left(\prod_{\mathrm{f} \in \mathrm{F}}\left(\mathbf{h}\left(\mathrm{l}_{\mathfrak{g}}(\mathrm{f})\right) \Rightarrow \mathbf{h}^{\prime}(\mathrm{f})\right) \Rightarrow \mathbf{h}^{\prime}\left(\left[\varphi^{\prime}\right]_{\mathfrak{M}}\right)\right) \quad\left(\text { by } \mathcal{H}_{4}\right) \\
& \geq \prod_{\mathbf{h}^{\prime} \in \mathfrak{S}^{\mathfrak{E}}}\left(\left(\mathbf{h}\left(\mathrm{l}_{\mathfrak{g}}\left(\left[\varphi^{\prime}\right]_{\mathfrak{M}}\right)\right) \Rightarrow \mathbf{h}^{\prime}\left(\left[\varphi^{\prime}\right]_{\mathfrak{M}}\right)\right) \Rightarrow \mathbf{h}^{\prime}\left(\left[\varphi^{\prime}\right]_{\mathfrak{M}}\right)\right) \quad\left(\text { fix } \mathrm{f}=\left[\varphi^{\prime}\right]_{\mathfrak{M}}, \mathcal{H}_{4}\right) \\
& \geq \prod_{\mathbf{h}^{\prime} \in \mathfrak{S}^{\mathfrak{E}}} \mathbf{h}\left(\mathrm{l}_{\mathfrak{g}}\left(\left[\varphi^{\prime}\right]_{\mathfrak{M}}\right)\right) \quad\left(\text { by } \mathcal{H}_{11}\right) \\
& =\mathbf{h}\left(\left[\square \varphi^{\prime}\right]_{\mathfrak{M}}\right)
\end{aligned}
$$

( $\square$, Part 2.) $v^{\mathfrak{E}}\left(\mathbf{h}, \square \varphi^{\prime}\right) \leq \mathbf{h}\left(\left[\square \varphi^{\prime}\right]_{\mathfrak{M}}\right)$

Towards a contradiction, suppose that for a fixed $\mathbf{h}_{0} \in \mathfrak{S}^{\mathfrak{E}}, v^{\mathfrak{E}}\left(\mathbf{h}_{0}, \square \varphi^{\prime}\right) \not \leq \mathbf{h}_{0}\left(\left[\square \varphi^{\prime}\right]_{\mathfrak{M}}\right)$. Let $\mathrm{T}$ be the following subset of $\mathrm{F}$ :

$$
\mathrm{T}=\left\{\left[\mathbf{h}_{0}\left(\mathrm{l}_{\mathfrak{g}}(\mathrm{g})\right)\right] \Rightarrow_{\mathrm{F}} \mathrm{g}, \mathrm{z} \Rightarrow_{\mathrm{F}}\left[\mathbf{h}_{0}\left(\mathrm{~m}_{\mathfrak{g}}(\mathrm{z})\right)\right]: \mathrm{g}, \mathrm{z} \in \mathrm{F}\right\} .
$$

Also let

$$
\mathbf{f}=\left[v^{\mathfrak{E}}\left(\mathbf{h}_{0}, \square \varphi^{\prime}\right)\right] \Rightarrow_{\mathrm{F}}\left[\varphi^{\prime}\right]_{\mathfrak{M}}
$$

Note that $v^{\mathfrak{E}}\left(\mathbf{h}_{0}, \square \varphi^{\prime}\right)$ is a member of $\mathcal{H}$ and thus $\left[v^{\mathfrak{E}}\left(\mathbf{h}_{0}, \square \varphi^{\prime}\right)\right]$ is a legitimate constant function in $\mathrm{F}$. We will first show that $\langle\emptyset, \mathrm{T}\rangle$ has the f-f.m.p.: by Lemma 4.26, it suffices to show that for every finite $\mathrm{T}_{0} \subseteq \mathrm{T}, \prod_{0} \mathrm{~T}_{0} \underline{\mathrm{F}}_{\mathrm{F}} \mathrm{f}$. If this was not the case, there would exist finite subsets $\left\{\mathrm{g}_{0}, \ldots, \mathrm{g}_{m}\right\}$ and $\left\{\mathrm{z}_{0}, \ldots, \mathrm{z}_{n}\right\}$ of $\mathrm{F}$, such that:

$$
\prod_{i=1, \ldots, m}\left(\left[\mathbf{h}_{0}\left(\mathrm{l}_{\mathfrak{g}}\left(\mathrm{g}_{i}\right)\right)\right] \Rightarrow_{\mathrm{F}} \mathrm{g}_{i}\right) \cdot \prod_{j=1, \ldots, n}\left(\mathrm{z}_{j} \Rightarrow_{\mathrm{F}}\left[\mathbf{h}_{0}\left(\mathrm{~m}_{\mathfrak{g}}\left(\mathrm{z}_{j}\right)\right)\right]\right) \leq_{\mathrm{F}}\left[v^{\mathfrak{E}}\left(\mathbf{h}_{0}, \square \varphi^{\prime}\right)\right] \Rightarrow_{\mathrm{F}}\left[\varphi^{\prime}\right]_{\mathfrak{M}}
$$

Combining Lemma 4.15[(1),(2),(4),(5)] and Fact 4.17 in one step, we obtain

$$
\prod_{i=1, \ldots, m}\left(\left[\mathbf{h}_{0}\left(\mathrm{l}_{\mathfrak{g}}\left(\mathrm{g}_{i}\right)\right)\right] \Rightarrow_{\mathrm{F}} \mathrm{l}_{\mathfrak{g}}\left(\mathrm{g}_{i}\right)\right) \cdot \prod_{j=1, \ldots, n}\left(\mathrm{~m}_{\mathfrak{g}}\left(\mathbf{z}_{j}\right) \Rightarrow_{\mathrm{F}}\left[\mathbf{h}_{0}\left(\mathrm{~m}_{\mathfrak{g}}\left(\mathbf{z}_{j}\right)\right)\right]\right) \leq_{\mathrm{F}}\left[v^{\mathfrak{E}}\left(\mathbf{h}_{0}, \square \varphi^{\prime}\right)\right] \Rightarrow_{\mathrm{F}}\left[\square \varphi^{\prime}\right]_{\mathfrak{M}} .
$$

Noting that $\mathbf{h}_{0}$ is $\mathcal{H}$-proper and homomorphisms are order-preserving, we apply $\mathbf{h}_{0}$ to each part of the inequality, to obtain that

$$
\prod_{i=1, \ldots, m}\left(\mathbf{h}_{0}\left(\mathrm{l}_{\mathfrak{g}}\left(\mathrm{g}_{i}\right)\right) \Rightarrow \mathbf{h}_{0}\left(\mathrm{l}_{\mathfrak{g}}\left(\mathrm{g}_{i}\right)\right)\right) \cdot \prod_{j=1, \ldots, n}\left(\mathbf{h}_{0}\left(\mathrm{~m}_{\mathfrak{g}}\left(\mathbf{z}_{j}\right)\right) \Rightarrow \mathbf{h}_{0}\left(\mathrm{~m}_{\mathfrak{g}}\left(\mathbf{z}_{j}\right)\right)\right) \leq v^{\mathfrak{E}}\left(\mathbf{h}, \square \varphi^{\prime}\right) \Rightarrow \mathbf{h}_{0}\left(\left[\square \varphi^{\prime}\right]_{\mathfrak{M}}\right),
$$


Hence,

$$
\top \leq v^{\mathfrak{E}}\left(\mathbf{h}_{0}, \square \varphi^{\prime}\right) \Rightarrow \mathbf{h}_{0}\left(\left[\square \varphi^{\prime}\right]_{\mathfrak{M}}\right)
$$

or equivalently

$$
v^{\mathfrak{E}}\left(\mathbf{h}_{0}, \square \varphi^{\prime}\right) \leq \mathbf{h}_{0}\left(\left[\square \varphi^{\prime}\right]_{\mathfrak{M}}\right)
$$

and we have reached a contradiction.

Now, we can extend $\langle\emptyset, T\rangle$ to a maximal $\left\langle S_{m}, T\right\rangle$ with the f-f.m.p., according to Theorem 4.23, and take $\mathbf{h}_{\mathrm{S}_{\mathrm{m}}}$ be the $\mathrm{f}$-compatible function corresponding to $\left\langle\mathrm{S}_{\mathrm{m}}, \mathbf{T}\right\rangle$. We will combine some facts to reach a contradiction:

$$
\begin{aligned}
v^{\mathfrak{E}}\left(\mathbf{h}_{0}, \square \varphi^{\prime}\right) & =\prod_{\mathbf{h} \in \mathfrak{S}^{\mathfrak{E}}}\left(\mathfrak{g}^{\mathfrak{E}}\left(\mathbf{h}_{0}, \mathbf{h}\right) \Rightarrow v^{\mathfrak{E}}\left(\mathbf{h}, \varphi^{\prime}\right)\right) \\
& \leq \mathfrak{g}^{\mathfrak{E}}\left(\mathbf{h}_{0}, \mathbf{h}_{\mathrm{S}_{\mathfrak{m}}}\right) \Rightarrow v^{\mathfrak{E}}\left(\mathbf{h}_{\left.\mathrm{S}_{\mathfrak{m}}, \varphi^{\prime}\right)}\right. \\
& =\mathfrak{g}^{\mathfrak{E}}\left(\mathbf{h}_{0}, \mathbf{h}_{\mathrm{S}_{\mathfrak{m}}}\right) \Rightarrow \mathbf{h}_{\mathrm{S}_{\mathfrak{m}}}\left(\left[\varphi^{\prime}\right]_{\mathfrak{M}}\right)
\end{aligned}
$$

and hence, by $\mathcal{H}_{1}$,

$$
\begin{aligned}
\mathfrak{g}^{\mathfrak{E}}\left(\mathbf{h}_{0}, \mathbf{h}_{\mathrm{S}_{\mathrm{m}}}\right) & \leq v^{\mathfrak{E}}\left(\mathbf{h}_{0}, \square \varphi^{\prime}\right) \Rightarrow \mathbf{h}_{\mathrm{S}_{\mathrm{m}}}\left(\left[\varphi^{\prime}\right]_{\mathfrak{M}}\right) \\
& =\mathbf{h}_{\mathrm{S}_{\mathrm{m}}}\left(\left[v^{\mathfrak{E}}\left(\mathbf{h}_{0}, \square \varphi^{\prime}\right)\right] \Rightarrow_{\mathrm{F}}\left[\varphi^{\prime}\right]_{\mathfrak{M}}\right) \\
& =\mathbf{h}_{\mathrm{S}_{\mathrm{m}}}(\mathbf{f}) .
\end{aligned}
$$

Also,

$$
\begin{aligned}
\mathfrak{g}^{\mathfrak{E}}\left(\mathbf{h}_{0}, \mathbf{h}_{\mathrm{S}_{\mathrm{m}}}\right) & =\prod_{\mathbf{z} \in \mathrm{F}}\left(\mathbf{h}_{\mathrm{S}_{\mathrm{m}}}(\mathrm{z}) \Rightarrow \mathbf{h}_{0}\left(\mathrm{~m}_{\mathfrak{g}}(\mathrm{z})\right)\right) \cdot \prod_{\mathfrak{g} \in \mathrm{F}}\left(\mathbf{h}_{0}\left(\mathrm{l}_{\mathfrak{g}}(\mathrm{g})\right) \Rightarrow \mathbf{h}_{\mathrm{S}_{\mathrm{m}}}(\mathrm{g})\right) \\
& =\prod_{\mathbf{z} \in \mathrm{F}} \mathbf{h}_{\mathrm{S}_{\mathrm{m}}}\left(\mathrm{z} \Rightarrow_{\mathrm{F}}\left[\mathbf{h}_{0}\left(\mathrm{~m}_{\mathfrak{g}}(\mathrm{z})\right)\right]\right) \cdot \prod_{\mathfrak{g} \in \mathrm{F}} \mathbf{h}_{\mathrm{S}_{\mathrm{m}}}\left(\left[\mathbf{h}_{0}\left(\mathrm{l}_{\mathfrak{g}}(\mathrm{g})\right)\right] \Rightarrow_{\mathrm{F}} \mathrm{g}\right) \\
& =\prod_{\mathrm{t} \in \mathrm{T}} \mathbf{h}_{\mathrm{S}_{\mathrm{m}}}(\mathrm{t})
\end{aligned}
$$

(1) and (2) imply:

$$
\prod_{t \in T} \mathbf{h}_{\mathrm{S}_{\mathrm{m}}}(\mathrm{t}) \leq \mathbf{h}_{\mathrm{S}_{\mathrm{m}}}(\mathrm{f})
$$

Since $\mathcal{H}$ is finite, then for a finite $\mathrm{T}_{0} \subseteq \mathrm{T}$ we infer that $\prod_{\mathrm{t} \in \mathrm{T}} \mathbf{h}_{\mathrm{S}_{\mathrm{m}}}(\mathrm{t})=\prod_{\mathrm{t} \in \mathrm{T}_{0}} \mathbf{h}_{\mathrm{S}_{\mathrm{m}}}(\mathrm{t})$, and thus $\prod_{t \in T_{0}} \mathbf{h}_{S_{m}}(t) \leq \mathbf{h}_{s_{m}}(f)$, which contradicts the fact that $\mathbf{h}_{S_{m}}$ is $\mathbf{f}$-compatible w.r.t. $T$.

Embedding an $\mathcal{H}$-model to its canonical extension. The key to this embedding is of course the set of the point-generated $\mathcal{H}$-proper functions. We report here that, as in the classical case, by identifying a state $\mathfrak{s}$ of a frame $\mathfrak{F}$ with its associated pointgenerated function $\pi_{\mathfrak{s}}, \mathfrak{F}$ can be embedded in its canonical extension $\mathfrak{F}^{\mathfrak{E}}$. Moreover, not only the accessibility function, but also modal truth remains intact with respect to the mapping $\mathfrak{s} \mapsto \pi_{\mathfrak{s}}$. 
Theorem 4.29 1. For every $\mathfrak{s}, \mathfrak{t}$ in $\mathfrak{S}, \mathfrak{g}(\mathfrak{s}, \mathfrak{t})=\mathfrak{g}^{\mathfrak{E}}\left(\pi_{\mathfrak{s}}, \pi_{\mathfrak{t}}\right)$

2. For every $\mathfrak{s} \in \mathfrak{S}$ and $\varphi \in L_{\square \diamond}^{\mathcal{H}}, v(\mathfrak{s}, \varphi)=v^{\mathfrak{E}}\left(\pi_{\mathfrak{s}}, \varphi\right)$

Theorem 4.30 Let $\mathfrak{F}=\langle\mathfrak{S}, \mathfrak{g}\rangle$ be an $\mathcal{H}$-frame, $\mathfrak{F}^{\mathfrak{E}}=\left\langle\mathfrak{S}^{\mathfrak{E}}, \mathfrak{g}^{\mathfrak{E}}\right\rangle$ its canonical extension and $X$ a formula of $L_{\square \diamond}^{\mathcal{H}}$. Then

$$
\mathfrak{F}^{\mathfrak{E}} \Vdash X \text { implies } \mathfrak{F} \Vdash X \text {. }
$$

Remark 4.31 [Algebraic semantics and general frames for mv-ML]. The structure $\mathrm{F}$ of Definition 4.13 is a Heyting Algebra with Operators, the bimodal generalization of a full complex algebra. Based on this, it is tempting to try to define an algebraic semantics for $\mathrm{mv}-\mathrm{ML}$. The operators $\mathrm{l}_{\mathfrak{g}}$ and $\mathrm{m}_{\mathfrak{g}}$ serve for the interpretation of the modal operators $\square$ and $\diamond$; Lemma 4.15[(4) \& (5)] asserts that the operators satisfy two basic axioms of the proof theory of mv-MLs (cf. [7]) and it is easy to verify that K. $\square(A \supset B) \supset(\square A \supset \square B)$ is satisfied as well. Everything seems perfect, with a sole exception: we have to guarantee in this semantics that every $t \in \mathcal{H}$ is interpreted on $[t]$ and thus $[t]$ implicitly becomes a part of the similarity type, if our intended 'algebraic' semantics are to be well-behaved ${ }^{6}$. In that way, the encoding of $\mathcal{H}$ in $L_{\square \diamond}^{\mathcal{H}}$ which provides a certain amount of flexibility for epistemic applications in Knowledge Representation (cf. the weak modal axioms in [18]), practically trivializes the most straightforward attempt to algebraize mv-MLs. However, we have gained a small profit. En route, we have set the tools for defining a decent notion of a general $\mathcal{H}$-frame. It is obvious now that such a structure consists of an $\mathcal{H}$-frame, accompanied by a subalgebra of $\mathrm{F}$. An $\mathcal{H}$-model built on a general $\mathcal{H}$-frame will then require that for each $P \in \Phi,[P]_{\mathfrak{M}}$ is an element of the subalgebra.

\section{Conclusion}

In this paper we have developed a part of the model theory of many-valued modal logics, focusing on the most important frame constructions, and their associated model versions. There exist several reasons for considering such a project worth pursuing.

An independent mathematical interest: it is natural to develop model-theoretic structures in a broader context and this usually assists in understanding things better in the classical case. Moreover, it allows for testing the robustness of techniques and constructions which is an additional indication for their formal value. Many-valued modal logics provide a good context for developing a unifying modal model theory with a strong algebraic flavor.

An interesting perspective on model theory: this family of logics embodies an interesting mix of lattice theory and logic, actually inherited from its semantics which combine Kripke intuitionistic and modal frames [7]. Thus, setting up the model theory of mvMLs imports some sophisticated techniques from algebra, at least if one is willing to

\footnotetext{
${ }^{6}$ The footnote in Def. 4.18 is also relevant here.
} 
accept that Universal Algebra and Lattice theory provide some level of sophistication in logical investigations. Actually we believe that mv-MLs provide a context in which the classical Chang \& Keisler equation "model theory = universal algebra + logic" [4] finds a very natural incarnation.

The potential for applications in AI provided by the equivalent multiple-expert semantics. Multi-agent systems research is at a premium nowadays, as emphasis is paid on situations with complex intelligent entities collaborating in heterogeneous environments. Applications include distributed systems, formal verification, etc. In that respect, mvMLs are useful items in the rich and varying collection of non-classical logics in AI.

\section{References}

[1] R. Balbes and Ph. Dwinger. Distributive Lattices. University of Missouri Press, 1974.

[2] P. Blackburn, M. de Rijke, and Y. Venema. Modal Logic. Number 53 in Cambridge Tracts in Theoretical Computer Science. Cambridge University Press, 2001.

[3] A. Chagrov and M. Zakharyashev. Modal Logic, volume 35 of Oxford Logic Guides. Oxford University Press, 1997.

[4] C. C. Chang and H. J. Keisler. Model Theory, volume 73 of Studies in Logic and the Foundations of Mathematics. North Holland, Amsterdam, 3rd edition, 1990.

[5] B. A. Davey and H. A. Priestley. Introduction to Lattices and Order. Cambridge University Press, 1990.

[6] M. C. Fitting. Many-valued modal logics. Fundamenta Informaticae, 15:235-254, 1991.

[7] M. C. Fitting. Many-valued modal logics II. Fundamenta Informaticae, 17:55-73, 1992.

[8] M. C. Fitting. Many-valued non-monotonic modal logics. In A. Nerode and M. Taitslin, editors, Logical Foundations of Computer Science - Tver'92, volume 620 of Lecture Notes in Computer Science, pages 139-150. Springer-Verlag, 1992.

[9] M. C. Fitting. Tableaus for many-valued modal logic. Studia Logica, 55:63-87, 1995 .

[10] R. Goldblatt. Varieties of complex algebras. Annals of Pure and Applied Logic, 44:173-242, 1989.

[11] R. Goldblatt. Algebraic Polymodal Logic: a survey. Logic Journal of the IGPL, 8(4):393-450, 2000. Special Issue on Algebraic Logic edited by István Németi and Ildikó Sain. 
[12] R. Goldblatt. Mathematical modal logic: a view of its evolution. To appear in: D. van Dalen, J. Dawson and A. Kanamori: A History of Mathematical Logic, December 2002. Draft available through http://www.mcs.vuw.ac.nz/ ${ }^{\sim}$ rob.

[13] R. Goldblatt and S. K. Thomason. Axiomatic classes in propositional modal logic. In J. Crossley, editor, Algebra and Logic, pages 162-173. Springer Verlag, 1974.

[14] J. Halpern, R. Harper, N. Immerman, Ph. G. Kolaitis, M. Y. Vardi, and V. Vianu. On the unusual effectiveness of Logic in Computer Science. Bulletin of Symbolic Logic, 7(2), 2001. Summary of AAAS and NSF/CISE symposia by the same title held on 1999 and 2001.

[15] C. D. Koutras. A catalog of weak many-valued modal axioms and their corresponding frame classes. Journal of Applied Non-Classical Logics, 13(1):47-72, 2003.

[16] C. D. Koutras, G. Koletsos, and S. Zachos. Many-valued modal non-monotonic reasoning: Sequential stable sets and logics with linear truth spaces. Fundamenta Informaticae, 38(3):281-324, 1999.

[17] C. D. Koutras, Ch. Nomikos, and P. Peppas. Canonicity and completeness results for many-valued modal logics. Journal of Applied Non-Classical Logics, 12(1):7-41, 2002 .

[18] C. D. Koutras and P. Peppas. Weaker axioms, more ranges. Fundamenta Informaticae, 51(3):297-310, 2002.

[19] C. D. Koutras and S. Zachos. Many-valued reflexive autoepistemic logic. Logic Journal of the IGPL, 8(1):33-54, 2000.

[20] M. Kracht. Tools and Techniques in Modal Logic, volume 142 of Studies in Logic and the Foundations of Mathematics. Elsevier, Amsterdam, 1999.

[21] H. Rasiowa and R. Sikorski. The Mathematics of Metamathematics. PWN - Polish Scientific Publishers, Warsaw, third edition, 1970.

[22] J. van Benthem. Canonical modal logics and ultrafilter extensions. Journal of Symbolic Logic, 44:1-8, 1980.

[23] Y. Venema. Logic and universal algebra: some basic connections. Course Notes for LUATCS'99, November 1999. Available through http://staff.science.uva.nl/〜yde. 